\title{
WestVirginiaUniversity
}

THE RESEARCH REPOSITORY @ WVU

West Virginia Agricultural and Forestry Experiment

Davis College of Agriculture, Natural Resources

Station Bulletins

And Design

$1-1-1906$

\section{Commercial fertilizers : complete report for 1906}

\author{
J. H. Stewart
}

B. H. Hite

Follow this and additional works at: https://researchrepository.wvu.edu/ wv_agricultural_and_forestry_experiment_station_bulletins

\section{Digital Commons Citation}

Stewart, J. H. and Hite, B. H., "Commercial fertilizers : complete report for 1906" (1906). West Virginia Agricultural and Forestry Experiment Station Bulletins. 108.

https://researchrepository.wvu.edu/wv_agricultural_and_forestry_experiment_station_bulletins/108 @ WVU. It has been accepted for inclusion in West Virginia Agricultural and Forestry Experiment Station Bulletins by an authorized administrator of The Research Repository @ WVU. For more information, please contact ian.harmon@mail.wvu.edu. 
Digitized by the Internet Archive in 2010 with funding from

Lyrasis Members and Sloan Foundation 


\section{WEST VIRGINIA UNIVERSITY \\ AGRICULTURAL EXPERIMENT STATION \\ MORGANTOWN, W. VA.}

BulletTIN IO8.

- December 3i, 1906.

Commercial Fertilizers

\section{COMPLETE REPORT \\ FiOR \\ 1906}

By J. H. STEWART AND B. H. HITE,

[The Bulletins and Reports of this Station will be malled Iree to any citizen of West Virginia upon written application. Address Director of Agricultural Experiment Station, Morgantown, W. Va.] 
Name of Regent.

HoN. C. M. BABB

Hon. J. B. FinLEY.

Hon. D. C. Gallagher

Hon. E. M. Grant...................... Vorgantown, W. Va.

Hon. C. E. HaworTH..............Huntington, W. Va.

Hon. C. P. McNell. ...............Wheeling, W. Va.

Hon. L. J. Williams............... Lewisburg, W. Va. Hon. T. P. JacoBs.............. New Martinsville, W. Va. Hon. J. R. TROTTER................ Buckhannon, W. Va.

President of the Board of Regents.......... R. TROTTER President of the University.............. B. Purinton. Treasurer .................... R. WHITEHILL

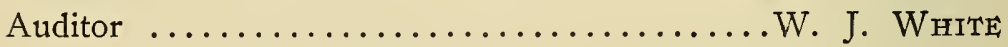

\section{STATION STAFF}

JAMES H. STEWART, A. M........ Director and Agriculturist BERT H. Hite, M. S.............Vice Director and Chemist John L. Sheldon, PH. D...............Plant Pathologist W. E. Rumsey, B. S. Agr. .......... Entomologist in Charge Horace Atwood, M. S. Agr. ...........Assistant Agriculturist T. C. Johnson, M. S...... Botanist and Associate Horticulturist Frank B. Kunst, A. B. ............... Assistant Chemist C. S. Forkum, B. S. .................. Assistant Chemist Leicester PatTon .................... Assistant Chemist

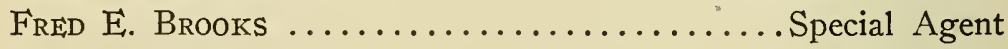

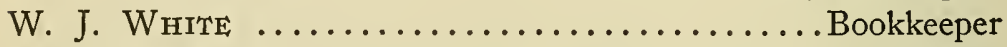
M. A. Stewart . . . . . . . . . . . . . . . . Librarian Alide EnGLE ......................... Stenographer 


\section{COMMERGIAL FERTILIZERS}

Complete Report for 1906. Bulletin 108

The following pages contain a complete report of the work done by this Experiment Station in accordance with the requirements of the Fertilizer Law of this State during the year ending December 3I, Ig06.

A list of the brands of fertilizers registered during the year and a list of the dealers will be found on the last pages of this report.

REMARKS. The attention of purchasers of commercial fertilizers is directed to the Remarks to be found at the close of the paragraphs devoted to brands that have fallen below the guarantee, and while many of these shortages are very small, all are inexcusable and a few (really a very few), are so large that farmers should consult their own interests by dealing with some other Company. 


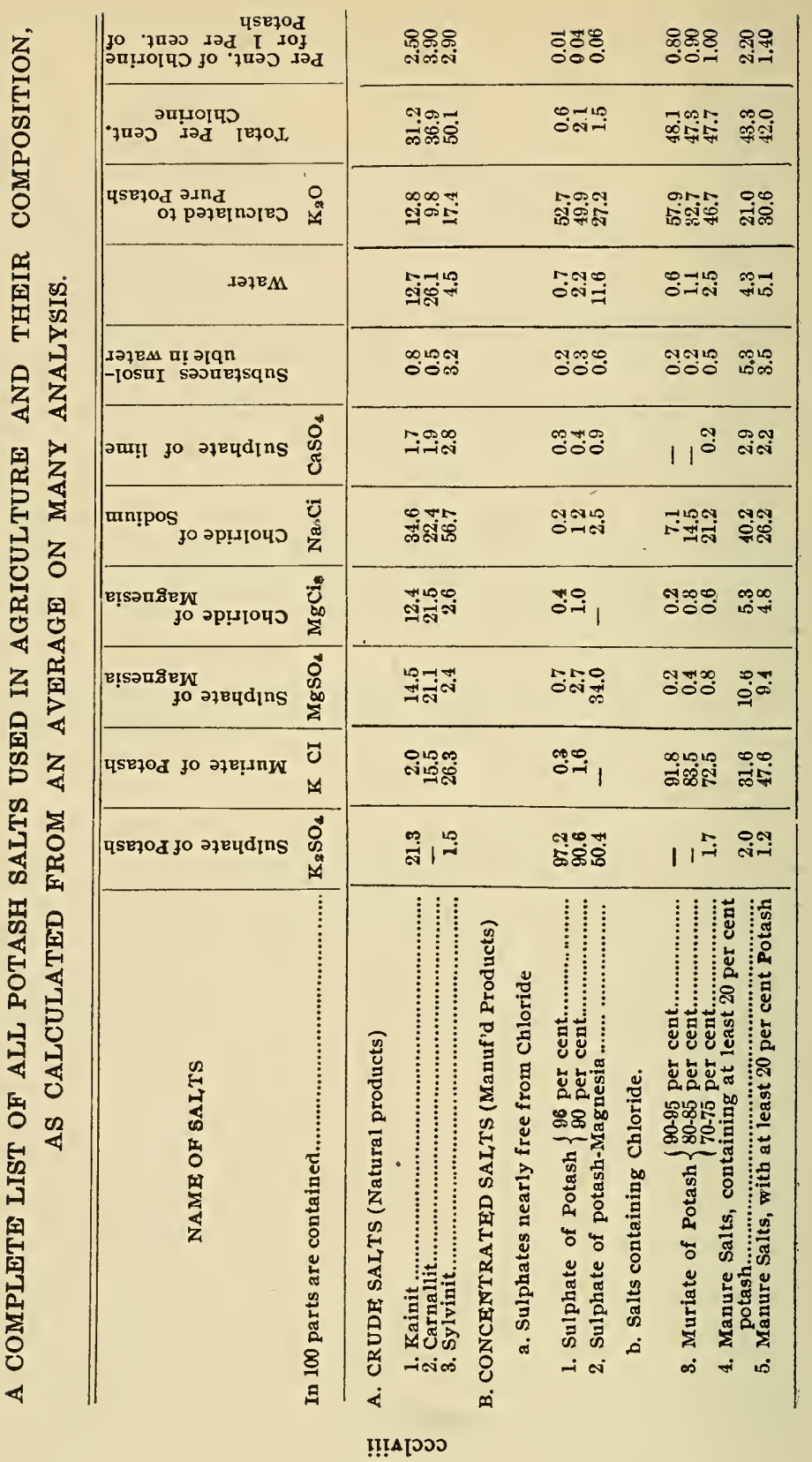




\title{
RESULTS OF THE
}

\section{Inspection of Commercial Fertilizers}

\author{
FOR I 906
}

THE

\section{AMERICAN AGRICULTURAL CHEMICAL COMPANY}

No. 26 Broadway, New York, and No. 208 N. Calvert STREET, BALTIMORE.

5455. A. A. C. CO'S BIG CROP PHOSPHATE. W. S. Corrothers, Agent, Catawba, W. Va. Guarantee: Phosphoric acid, soluble 6; reverted, 2 ; insoluble, I ; total, 9; available, 8; potash, 5 ; phosphoric acid from dissolved phosphate rock; potash from manure salt. Found: Phosphoric acid, soluble, 4:16; reverted, 5.10; insoluble, 0.55 ; total, 9.8I; available, 9.26; potash, $5.63 ;{ }^{*}$ chlorine, I.2.

5610. A. A. C. CO'S BIG CROP PHOSPHATE. Exchange Mill Co., Agent, Grafton, W. Va. Guarantee: (As above.) Found: Phosphoric acid, soluble, 4.46; reverted, 3.03 ; insoluble, 0.42 ; total, 7.91 ; available, 7.49; potash, 4.75 ; chlorine, 2.8 .

Remark: Source of potash equivalent to kainit.

56r6. A. A. C. CO'S BIG CROP PHOSPHATE. W. S. Corrothers, Agent, Little Falls, W. Va. Guarantee: (As above.) Found: Phosphoric acid, soluble, $2: 7 \mathrm{I}$; reverted, $6: 24$; insoluble, 0.70 ; total, 9.65 ; available, 8.95 ; potash, 5.64 ; chlorine, I.7.

* This represents the proportion of chlorine corresponding to one per cent. of potash in the fertilizer. The actual percentage of chlorine may be found by multiplying the above by the actual percentage of potash present. Chlorine is a constituent of muriate of potash, of common salt and of certain compounds of lime and magnesia. When high-grade sulphate is used as the source of potash the fertilizer should contain no more than traces of chlorine. The quantity of the latter increases directly with the inferiority of the source employed and its estimation serves approximately as an indication of such source. Where kainit is guaranteed and the chlorin $\theta$ exceeds 4.5 , it may be assumed that an inferior grade of this material has been used. See Page 358. 
56I9. A. A. C. CO'S BIG CROP PHOSPHATE. Johnson \& Gwinn, Agent, Alderson, W. Va. Guarantee: (As above.) Found: Phosphoric acid, soluble, 3.22 ; reverted, 4.01 ; insoluble, 0.52 ; total, 7.75 ; available, 7.23 ; potash, 5.94 ; chlorine, 1.7.

5621. A. A. C. CO'S BIG CROP PHOSPHATE. Curry \& Rogers, Agent, Marlinton, W. Va. Guarantee: (As above.) Found: Phosphoric acid, soluble, 4.35; reverted, 3.76; insoluble, o.78; total, 8.89; available, 8.II ; potash, 3.97 ; chlorine, 4.I.

5624. A. A. C. CO'S BIG CROP PHOSPHATE. H. E. Sharp, Agent, Alderson, W. Va. Guarantee: (As above.) Found: Phosphoric acid, soluble, 3.45; reverted, 4.39; insoluble, o.88; total, 8.72 ; available, 7.94 ; potash, 5.38 ; chlorine, 2.4 .

5635. A. A. C. CO'S BIG CROP PHOSPHATE. W. S. Corrothers, Agent, Little Falls, W. Va. Guarantee: (As above.) Found: Phosphoric acid, soluble, 4.80; reverted, 3.I4; insoluble, 0.59 ; total, 8.53 ; available, 7.94 ; potash, 5.1 I ; chlorine, 2.8 .

Remark: Source of potash equivalent to kainit.

5718. A. A. C. CO'S BIG CROP PHOSPHATE. J. M. Miller \& Bro., Agent, Ronceverte, W. Va. Guarantee: (As above.) Found: Phosphoric acid, soluble, 3.6I; reverted, 4.49; insoluble, I.70; total, 9.80; available, 8.10; potash, 5.14; chlorine, 2.4 .

5733. A. A. C. CO'S BIG CROP PHOSPHATE. Johnson \& Gwinn, Agent, Alderson, W. Va. Guarantee: (As above.) Found: Phosphoric acid, soluble, 4.22; reverted, 4.4I ; insoluble, 0.90; total, 9.53; available, 8.63; potash, 4.94; chlorine, 2.7 . Remark: Source of potash equivalent to kainit.

5734. A. A. C. CO'S BIG CROP PHOSPHATE. J. W. Hedrick, Agent, Alderson, W. Va. Guarantee: (As above.) Found: Phosphoric acid, soluble, 5.82 ; reverted, 3.08 ; insoluble, 0.77 ; total, 9.67 ; available, 8.90 ; potash, 4.92 ; chlorine, 2.8 .

Remark: Source of potash equivalent to kainit. 
548x. A. A. C. CO'S REGULAR CORN FERTILIZER. J. M. Miller \& Bro., Agent, Ronceverte, W. Va. Guarantee: Phosphoric acid, soluble, 4; reverted, 2 ; insoluble, I; total, 7 ; available, 6; potash, 3.00; phosphoric acid from dissolved phosphate rock; potash, from kainit. Found: Phosphoric acid, soluble, 2.5I ; reverted, 3.36; insoluble, O.4I ; total, 6.28; available, 5.87; potash, 2.96; chlorine, I.9.

5568. A. A. C. CO'S REGULAR CORN FERTILIZER, W. S. Stout, Agent, Harrisville, W. Va. Guarantee: (As above.) Found: Phosphoric acid, soluble, I.60; reverted, 4.86; insoluble, $0.4 \mathrm{I}$; total, 6.87 ; available, 6.46 ; potash, $3.3 \mathrm{I}$; chlorine, 7.I. Remark: Chlorine excessive.

5539. A. A. C. CO'S REGULAR CORN FERTILIZER. Cicero Phillips, Agent, Belington, W. Va. Guarantee: (As above.) Found: Phosphoric acid, soluble, I.56; reverted, 4.49; insoluble, 0.77 ; total, 6.82 ; available, 6.05; potash, 4.09; chlorine, 2.I.

5646. A. A. C. CO'S FINE GROUND BONE. Orie Carmen, Agent, Wellsburg, W. Va. Guarantee: Phosphoric acid, total, 22.80; nitrogen, 2.47; phosphoric acid from animal bone; nitrogen from animal bone. Found: 'Phosphoric acid, total, 22.96; nitrogen, 2.98; availability of nitrogen, *86.

*The figure for the availability of nitrogen represents that proportion of the total nitrogen of the fertilizer which is immediately available to the plant as food. The test employed is a modification of Street's neutral permaganate method. While the results obtained can be considered as but an approximation to the true values as indicated by actual field tests, nevertheless they are valuable as serving to show the relative availability of the nitrogen in various brands of fertilizers. An availability of 85 or above is considered high, $80-85$ medium, below 70 low. The availability of nitrogen in bone meals is nacessarily higher than in mixed goods unless nitrate is used in the latter. 
5647. A. A. C. CO'S FINE GROUND BONE. W. S. Corrothers, Agent, Catawba, W. Va. Guarantee: (As above.) Found: Phosphoric acid, total, 23.74; nitrogen, 2.40; availability of nitrogen, 94 .

5700. A. A. C. CO'S FINE GROUND BONE. Siever Hardware Co., Agent, Keyser, W. Va. Guarantee: (As above.) Found: Phosphoric acid, total, 23.59; nitrogen, 2.63; availability of nitrogen, 94 .

5688. A. A. C. CO'S PURE GROUND BONE. Thomas Nuzum, Agent, Grafton, W. Va. Guarantee: Phosphoric acid, total, 20.60; nitrogen, 3.30; phosphoric acid from animal bone; nitrogen from animal bone. Found: Phosphoric acid, total, 22.43; nitrogen, 3.23; availability of nitrogen, 72.

5633. A. A. C. CO'S PURE GROUND BONE. W. S. Corrothers, Agent, Little Falls, W. Va. Guarantee: (As above.) Found: Phosphoric acid, total, 22.11 ; nitrogen, 3.62 ; availability of nitrogen, 86 .

5682. A. A. C. CO'S KAINIT. Washington \& Alexander, Agent, Charles Town, W. Va. Guarantee: Potash, I2. Found: Potash, I4.70; chlorine, 2.6.

5732. A. A. C. CO'S GEM ALKALINE PHOSPHATE. J. W. Hedrick, Agent, Alderson, W. Va. Guarantee: Phosphoric acid, soluble, 4 ; reverted, 2 ; insoluble, I ; total, 7 ; available, 6; potash, 3; phosphoric acid from dissolved phosphate rock; potash from kainit. Found: Phosphoric acid, soluble, 3.7I ; reverted, 4.02 ; insoluble, 0.84 ; total, 8.57 ; available, 7.73; potash, 3.05 ; chlorine, 3.7 .

Remark: Chlorine excessive.

5772. A. A. C. CO'S GEM ALKALINE PHOSPHATE. R. Hunter, Agent, Berkeley Springs, W. Va. Guarantee: (As above.) Found: Phosphoric acid, soluble, 3.90; reverted, I.80; 
insoluble, I.I4; total, 6.84; available, 5.70; potash, 3.04 ; chlorine, 4.5 .

Remark: Chlorine excessive.

578I. A. A. C. CO'S GEM ALKALINE PHOSPHATE. T. B. Drummond \& Co., Agent, Buckhannon, W. Va. Guarantee: (As above.) Found: Phosphoric acid, soluble, I.47; reverted, 5.57 ; insoluble, I.22 ; total, 8.26; available, 7.04 ; potash, 3.63 ; chlorine, 2.5 .

5695. A. A. C. CO'S GEM ALKALINE PHOSPHATE. Belington Ice \& Bottling Co., Agent, Belington, W. Va. Guarantee: (As above.) Found: Phosphoric acid, soluble, 4.03; reverted, $2.6 \mathrm{I}$; insoluble, 0.50 ; total, 7.14 ; available, 6.64 ; potash, 3.43; chlorine, 3.9.

Remark: Chlorine excessive.

57OI. A.'A. C. CO'S GEM ALKALINE PHOSPHATE. Siever Hardware Co., Agent, Keyser, W. Va. Guarantee: (As above.) Found: Phosphoric acid, soluble, 4.6I; reverted, 2; insoluble, 0.46 ; total, 7.07 ; available, $6.6 \mathrm{I}$; potash, 3.48 ; chlorine, 4 .

Remark: Chlorine excessive.

55I8. A. A. C. CO'S SUPERPHOSPHATE. Philippi Mill Co., Philippi, W. Va. Guarantee: Phosphoric acid, soluble, I4; reverted, 2 ; insoluble, I ; total, I7 ; available, I6; phosphoric acid from dissolved phosphate rock. Found: Phosphoric acid, soluble, 14.07; reverted, 2.08; insoluble, 0.17; total, I6.32; available, I6.I5.

5579. A. A. C. CO'S BONE MEAL. West Virginia Implement Co., Agent, Elkins, W. Va. Guarantee: Phosphoric acid, total, I3.75; nitrogen, I.65; phosphoric acid from animal bone; nitrogen from animal bone. Found: Phosphoric acid, total, I4.97; nitrogen, I.68; availability of nitrogen, 86.

573I. BRADLEY'S SOLUBLE DISSOLVED BONE. J. W. Hedrick, Agent, Alderson, W. Va. Guarantee: Phosphoric 
acid, soluble, I2 ; reverted, 2 ; insoluble, I ; total, 15 ; available, I4; phosphoric acid from dissolved phosphate rock. Found: Phosphoric acid, soluble, II.64; reverted, 3.41 ; insoluble, 0.47 ; total, $\mathrm{x} 5.52$; available, 15.05 .

5730. BRADLEY'S NIAGARA PHOSPHATE. J. W. Hedrick, Agent, Alderson, W. Va. Guarantee: Phosphoric acid, soluble, 5 ; reverted, 2 ; insoluble, I ; total, 8; available, 7 ; nitrogen, 82 ; potash, I; phosphoric acid from dissolved animal bone and dissolved phosphate rock; potash from kainit; nitrogen not less than one-tenth from bone tankage or not less than onetenth from fish, the remainder from base goods, made from animal matter, treated by our own process. Found: Phosphoric acid, soluble, 4.54; reverted, 4.16; insoluble, I.52; total, I0.22; available, 8.70 ; nitrogen, 0.95 ; potash, I. 57 ; availability of nitrogen, 93 ; chlorine, 2.7 .

5573. BRADLEY'S BEAN AND POTATO. O. R. Carmen, Agent, Wellsburg, W. Va. Guarantee: Phosphoric acid, soluble, 6 ; reverted, 2 ; insoluble, I ; total, 9 ; available, 8 ; nitrogen, 0.82 ; potash, 4 ; phosphoric acid from dissolved animal bone and dissolved phosphate rock; nitrogen not less than one-tenth from bone tankage or not less than one-tenth from fish, the remainder from base goods made from animal matter, treated by our own process; potash $1 / 4$ to $3 / 4$ from manure salts, $1 / 4$ to $3 / 4$ from kainit. Found: Phosphoric acid, soluble, 5.65; reverted, 2.52 ; insoluble, 0.70; total, 8.87 ; available, 8.17 ; nitrogen, 0.97 ; potash, 4.29; availability of nitrogen, 82 ; chlorine, I.

5477. CANTON CHEMICAL POTATO MANURE. J. M. Miller \& Bro., Agent, Ronceverte, W. Va. Guarantee: Phosphoric acid, soluble, 4 ; reverted, 2 ; insoluble, I ; total, 7 ; available, 6; nitrogen, I.24; potash, 5 ; phosphoric acid from dissolved animal bone and dissolved phosphate rock; nitrogen not less than one-tenth from bone tankage, not less than one-tenth from fish, the remainder from base goods, made from animal matter, treated by our own process; potash $1 / 4$ to $3 / 4$ from manure salt and $1 / 4$ to $3 / 4$ from kainit. Found: Phosphoric acid, soluble, 
4.03 ; reverted, 2.42 ; insoluble, 0.75 ; total, 7.20 ; available, 6.45 ; nitrogen, I.43; potash, 4.58; availability of nitrogen, 92; chlorine, 0.7 .

Remark: Potash low.

55II. CANTON CHEMICAL POTATO MANURE. R. W. Sovine, Agent, Hurricane, W. Va. Guarantee: (As above.) Found: Phosphoric acid, soluble, 2.28; reverted, 4.01 ; insoluble, 2.02 ; total, 8.3I ; available, 6.29; nitrogen, I.52; potash, 5.53; availability of nitrogen, 77 ; chlorine, I.I.

5567. CANTON CHEMICAL, POTATO MANURE. Dean \& Reeves, Agent, Fairmont, W. Va. Guarantee: (As above.) Found: Phosphoric acid, soluble, 2.88; reverted, 3.46; insoluble, I.II ; total, 7.45; available, 6.34; nitrogen, I.I9; potash, 4.82; availability of nitrogen, 78 ; chlorine, I.4.

5576. CANTON CHEMICAL POTATO MANURE. West Virginia Implement Co., Agent, Elkins, W. Va. Guarantee: (As above.) Found: Phosphoric acid, soluble, I.O2; reverted, 4.9I ; insoluble, I.57; total, 7.50; available, 5.93; nitrogen, I.4I ; potash, 4.72 ; availability of nitrogen, 85 ; chlorine, 2.I.

5588. CANTON CHEMICAL POTATO MANURE. Buckhannon Grocery Co., Agent, Buckhannon, W. Va. Guarantee: (As above.) Found: Phosphoric acid, soluble, 2.74; reverted, 3.48; insoluble, I.07 ; total, 7.29; available, 6.22 ; nitrogen, I.23; potash, 5.42; availability of nitrogen, 86 ; chlorine, 2.4 .

5478. CANTON CHEMICAL BAKER'S DISSOLVED S. C. BONE. J. M. Miller \& Bro., Agent, Ronceverte, W. Va. Guarantee: Phosphoric acid, soluble, 12; reverted, 2 ; insoluble, I ; total, I5 ; available, I4; phosphoric acid from dissolved phosphate rock. Found: Phosphoric acid, soluble, II.77; reverted, 3.65 ; insoluble, 0.55 ; total, "I 5.97 ; available, $\mathbf{I} 5.42$.

5550. CANTON CHEMICAL BAKER'S DISSOLVED S. C. BONE. T. P. Licklider, Agent, Martinsburg, W. Va. Guar- 
antee: (As above.) Found: Phosphoric acid, soluble, I0.30; reverted, 3.84 ; insoluble, 0.69 ; total, I4.83; available, I4.I4.

5587. CANTON CHEMICAL BAKER'S DISSOLVED S. C. BONE. Buckhannon Grocery Co., Agent, Buckhannon, W. Va. Guarantee: (As above.) Found: Phosphoric acid, soluble, II.I7 ; reverted, 4.I2 ; insoluble, 0.93; total, I6.22 ; available, I 5.29.

5702. CANTON CHEMICAL BAKER'S DISSOLVED S. C. BONE. Siever Hardware Co., Agent, Keyser, W. Va. Guarantee: (As above.) Found: Phosphoric acid, soluble, I2.I I ; reverted, 3.57 ; insoluble, 0.38; total, I6.06; available, 15.68 .

5479. CANTON CHEMICAL BAKER'S SPECIAL WHEAT, CORN AND GRASS MIXTURE. J. M. Miller \& Bro., Agent, Roncevere, W. Va. Guarantee: Phosphoric acid, soluble, 7 ; reverted, 2 ; insoluble, I ; total, ıo ; available, 9 ; nitrogen, 0.82 ; potash, 2 ; phosphoric acid from dissolved animal bone and dissolved phosphate rock; nitrogen, not less than one-tenth from bone tankage, or not less than one-tenth from fish, the remainder from base goods, made from animal matter, treated by our own process; potash from kainit. Found: Phosphoric acid, soluble, 6.I5; reverted, 3.19 ; insoluble, o.82; total, Io.I6; available, 9.34 ; nitrogen, $0.7 \mathrm{I}$; potash, 2.03; availability of nitrogen, 87 ; chlorine, 3.9 .

Remark: Nitrogen low; chlorine excessive.

5586. CANTON CHEMICAL BAKER'S SPECIAL WHEAT, CORN AND GRASS MIXTURE. Buckhannon Grocery Co., Agent, Buckhannon, W. Va. Guarantee: (As above.) Found: Phosphoric acid, soluble, 2.97; reverted, 6.66; insoluble, I.05; total, I0.68; available, 9.63; nitrogen, I.02; potash, 2.24 ; availability of nitrogen, 76 ; chlorine, $\mathbf{I}$.

5672. CANTON CHEMICAL BAKER'S SPECIAL WHEAT, CORN AND GRASS MIXTURE. Washington \& 
Alexander, Agent, Charles Town, W. Va. Guarantee: (As above.) Found: Phosphoric acid, soluble, 6.65 ; reverted, 2.44; insoluble, 1.04; tota1, I0.13; available, 9.09; nitrogen, 0.90; potash, 2.37; availability of nitrogen, 70 ; chlorine, I.5.

5703. CANTON CHEMICAL BAKER'S SPECIAL WHEAT, CORN AND GRASS MIXTURE. Siever Hardware Co., Keyser, W. Va. Guarantee: (As above.) Found: Phosphoric acid, soluble, 6.52; reverted, 2.27 ; insoluble, I.38; total, I0.17; available, 8.79; nitrogen, 89; potash, 2.02; availability of nitrogen, 72 ; chlorine, I. 8 .

5779. CANTON CHEMICAL BAKER'S SPECIAL WHEAT, CORN AND GRASS MIXTURE. Upshur Grocery Co., Agent, Buckhannon, W. Va. Guarantee: (As above.) Found: Phosphoric acid, soluble, 7.04; reverted, 0.62 ; insoluble, 2.46; total, 10.12 ; available, 7.66; nitrogen, 97 ; potash, 2.36; availability of nitrogen, 80 ; chlorine, 2.3 .

Remark: Available phosphoric acid low.

5577. CANTON ..CHEMICAL ..BAKER'S SPECIAL, WHEAT, CORN AND GRASS MIXTURE. W. Va. Implement Co., Agent, Elkins, W. Va. Guarantee: (As above.) Found: Phosphoric acid, soluble, 4.95; reverted, 3.32; insoluble, 1.06; total, 9.33; available, 8.27 ; nitrogen, 1.68; potash, 2.39; availability of nitrogen, 80 ; chlorine, 2.4 .

555I. CANTON CHEMICAL SOLUBLE BONE AND POTASH. T. P. Licklider, Agent, Martinsburg, W. Va. Guarantee: Phosphoric acid, soluble, 8; reverted, 2; insoluble, I ; total, II; available, Io; potash, 2; phosphoric acid from dissolved phosphate rock; potash from kainit. Found: Phosphoric acid, soluble, 6.04 ; reverted, 3.67 ; insoluble, 0.61 ; total, Io.32; available, $9.7 \mathrm{I}$; potash, 2.63 ; chlorine, 3.3.

Remark: Available phosphoric acid low; chlorine excessive.

5589. CANTON CHEMICAL SOLUBLE BONE AND POTASH. Buckhannon Grocery Co., Agent, Buckhannon, W. 
Va. Guarantee: (As above.) Found: Phosphoric acid, soluble, 5.83 ; reverted, 4.40 ; insoluble, 0.49 ; total, 10.72; available, 10.23; potash, 2.36; chlorine, I.9.

57II. CANTON CHEMICAL SOLUBLE BONE AND POTASH. Siever Hardware Co., Agent, Keyser, W. Va. Guarantee: (As above.) Found: Phosphoric acid, soluble, 8.5I ; reverted, 2.54; insoluble, 0.36; total, II.4I ; available, II.05; potash, 2.27 ; chlorine, 2.8 .

5780. CANTON CHEMICAL SOLUBLE BONE AND $P O T A S H$. Upshur Grocery Co., Agent, Buckhannon, W. Va. Guarantee: (As above.) Found: Phosphoric acid, soluble, 7.68; reverted, $2.5 \mathrm{I}$; insoluble, 0.9I ; total, II.IO; available, I0.19; potash, 2.19; chlorine, 2.9.

5559. CANTON CHEMICAL GAME GUANO. Dean \& Reeves, Agent, Fairmont, W. Va. Guarantee: Phosphoric acid, soluble, 6; reverted, 2 ; insoluble, 1 ; total, 9; available, 8; nitrogen, I.65; potash, 2 ; phosphoric acid from dissolved animal bone and dissolved phosphate rock; nitrogen not less than onetenth from bone tankage, not less than one-tenth from fish, the remainder from base goods, made from animal matter, treated by our own process; potash from kainit. Found: Phosphoric acid, soluble, 4.39; reverted, 3.80 ; insoluble, I.30; total, 9.49; available, 8.19; nitrogen, I.59; potash, 2.09; availability of nitrogen, 89; chlorine, 3.2 .

Remark: Chlorine excessive.

55IO. CLEVELAND DRYER HORSEHEAD PHOSPHATE. R. W. Sovine, Agent, Hurricane, W. Va. Guarantee: Phosphoric acid, soluble, 8; reverted, 2; insoluble, I ; total, II ; available, IO; phosphoric acid from dissolved phosphate rock. Found: Phosphoric acid, soluble, 5.33; reverted, 5.74; insoluble, 3.19; total, I4.26; available, I I.07.

55I2. CLEVELAND DRYER HORSEHEAD PHOSPHATE AND POTASH. R. W. Sovine, Agent, Hurricane, 
W. Va. Guarantee: Phosphoric acid, soluble, 8; reverted, 2; insoluble, I ; total, Ir ; available, Io; potash, 2; phosphoric acid from dissolved phosphate rock; potash from kainit. Found: Phosphoric acid, soluble, 3.53; reverted, 6.4I ; insoluble, 3.49; total, I3.43; available, 9.94 ; potash, 2.73 ; chlorine, .8.

5452. DETRICK'S QUICKSTEP BONE PHOSPHATE. W. S. Corrothers, Agent, Cattawba, W. Va. Guarantee: Phosphoric acid, soluble, 6; reverted, 2 ; insoluble, I ; total, 9; available, 8 ; nitrogen, 2.47 ; potash, 4 ; phosphoric acid from dissolved animal bone and dissolved phosphate rock; nitrogen not less than onetenth from bone tankage, not less than one-tenth from fish, the remainder from base goods, made from animal matter, treated by our own process; potash from muriate of potash. Found: Phosphoric acid, soluble, 7.29; reverted, 2.48; insoluble, 0.63; total, ro.40; available, 9.77 ; nitrogen, 2.35 ; potash, 4.60 ; availability of nitrogen, 87 ; chlorine, 1 .

Remark: Nitrogen low.

56I4. DETRICK'S QUICKSTEP BONE PHOSPHATE. W. S. Corrothers, Agent, Little Falls, W. Va. Guarantee: (As above.) Found: Phosphoric acid, soluble, 6.37; reverted, 2.68; insoluble, $0.5 \mathrm{I}$; total, 9.56; available, 9.05 ; nitrogen, 2.40 ; potash, 4.07; availability of nitrogen, 79 ; chlorine, r.6.

5453. DETRICK'S DISSOLVED S. C. BONE. W. S. Corrothers, Agent, Catawba, W. Va. Guarantee: Phosphoric acid, soluble, I2; reverted, 2; insoluble, I; total, I5; available, I4; phosphoric acid from dissolved phosphate rock. Found: Phosphoric acid, soluble, 9.89; reverted, 5.89; insoluble, 0.75; total, 16.53 ; available, 15.68 .

5454. DETRICK'S CORN AND OATS FERTILIZER. W. S. Corrothers, Agent, Little Falls, W. Va. Guarantee: Phosphoric acid, soluble, 7 ; reverted, 2 ; insoluble, $\mathrm{r}$; total, ro; available, 9 ; nitrogen, 0.82 ; potash, 3 ; phosphoric acid from dissolved animal bone and dissolved phosphate rock; nitrogen not less than one-tenth from bone tankage or not less than one-tenth from fish, 
the remainder from base goods, made from animal matter, treated by our own process; potash $1 / 4$ to $3 / 4$ from manure salt and $1 / 4$ to 3/4 from kainit. Found: Phosphoric acid, soluble, 7.75 ; reverted, 2.73 ; insoluble, 0.83 ; total, II.3I; available, I0.48; nitrogen, I.03; potash, 3.59 ; availability of nitrogen, 77 ; chlorine, I.2.

5456. DETRICK'S SOLUBLE BONE PHOSPHATE AND POTASH. W. S. Corrothers, Agent, Little Falls, W. Va. Guarantee: Phosphoric acid, soluble, 8; reverted, 2; insoluble, I; total, II; available, IO; potash, 2; phosphoric acid from dissolved phosphate rock; potash from kainit. Found: Phosphoric acid, soluble, 3.43; reverted, 7.52; insoluble, 1.23; total, I2.I8; available, I0.95; potash, 3.08; chlorine, I.3.

56I7. DETRICK'S SOLUBLE BONE PHOSPHATE AND POTASH. W. S. Corrothers, Agent, Little Falls, W. Va. Guarantee: (As above.) Found: Phosphoric acid, soluble, 2.88; reverted, 7.35; insoluble, 1.16; total, II.39; available, 10.23; potash, 3.37 ; chlorine, 1.9.

5648. DETRICK'S SOLUBLE-BONE PHOSPHATE AND POTASH. W. S. Corrothers, Agent, Catawba, W. Va. Guarantee: (As above.) Found: Phosphoric acid, soluble, 7.42 ; reverted, 3.07 ; insoluble, .38; total, 10.87; available, 10.49; potash, 2.01 ; chlorine, 3 .

547I. DETRICK'S KANGAROO KOMPLETE KOMPOUND. W. S. Corrothers, Agent, Little Falls, W. Va. Guarantee: Phosphoric acid, soluble, 6; reverted, 2 ; insoluble, 1 ; total, 9 ; available, 8 ; nitrogen, 1.65 ; potash, 3 ; phosphoric acid from dissolved animal bone and dissolved phosphate rock; nitrogen, not less than one-tenth from bone tankage, not less than one-tenth from fish, the remainder from base goods, made from animal matter, treated by our own process; potash, $1 / 4$ to $3 / 4$ from manure salt and $1 / 4$ to $3 / 4$ from kainit. Found: Phosphoric acid, soluble, 4.07; reverted, 6.03; insoluble, 1.52; total, Ir.62; available, Io.1o; nitrogen, I.62; potash, 3.32 ; availability of nitrogen, 85 ; chlorine, 1.3 . 
57I7. DETRICK'S KANKAROO KOMPLETE .KOMPOUND. J. M. Miller \& Bro., Agent, Ronceverte, W. Va. Guarantee: (As above.) Fiound: Phosphoric acid, soluble, 5.05 ; reverted, 3.56 ; insoluble, I.89; total, I0.50; available, 8.6I ; nitrogen, I.54; kainit, 2.82 ; availability of nitrogen, 92 ; chlorine, 2.7 .

5623. DETRICK'S KANGAROO KOMPLETE KOMPOUND. J. M. Miller \& Bro., Agent, Ronceverte, W. Va. Guarantee: (As above.) Found: Phosphoric acid, soluble, 3.95; reverted, 3.93; insoluble, 1.29; total, 9.I7; available, 7.88; nitrogen, I.53; potash, 3.2I ; availability of nitrogen, 89; chlorine, 2.

Remark: Nitrogen low.

55'5. DETRICK'S KANGAROO KOMPLETE KOMPOUND. W. N. McCallister, Agent, Hurricane, W. Va. Guarantee: (As above.) Found: Phosphoric acid, soluble, 4.23; reverted, 4.13 insoluble, 2.17 ; total, ro.53; available, 8.36 ; nitrogen, 2.13 ; potash, 3.08 ; availability of nitrogen, 85 ; chlorine, 2.7 .

5472. DETRICK'S PARAGON AMMONIATED BONE PHOSPHATE WITH POTASH. W. S. Corrothers, Agent, Little Falls, W. Va. Guarantee: Phosphoric acid, soluble, 5; reverted, 2 ; insoluble, I ; total, 8 ; available, 7 ; nitrogen, .82; potash, I; phosphoric acid from dissolved animal bone and dissolved phosphate rock; nitrogen, not less than one-tenth from bone tankage, or not less than one-tenth from fish, the remainder from base goods, made from animal matter, treated by our own process. Found: Phosphoric acid, soluble, 6.40; reverted, 2.88; insoluble, I.Io; total, Io.38; available, 9.28 ; nitrogen, I.33; potash, I.09; availability of nitrogen, 76 ; chlorine, 3 .

5637. DETRICK'S PARAGON AMMONIATED BONE PHOSPHATE WITH POTASH. W. S. Corrothers, Agent, Little Falls, W. Va. Guarantee: (As above.) Found: Phosphoric acid, soluble, 4.48; reverted, $3 . \mathrm{II}$; insoluble, I.20; total, 
8.79; available, 7.59 ; nitrogen, .80 ; potash, I.40; availability of nitrogen, 64 ; chlorine, 5 .

Remark: Chlorine excessive.

55I4. DETRICK'S IMPERIAL COMPOUND. W. S. McCallister, Agent, Hurricane, W. Va. Guarantee: Phosphoric acid, soluble, 6; reverted, 2 ; insoluble, I ; total, 9 ; available, 8 ; nitrogen, 82; potash, 2 ; phosphoric acid from dissolved animal bone and dissolved phosphate rock; nitrogen, not less than onetenth from bone tankage, or not less than one-tenth from fish, the remainder from base goods, made from animal matter, treated by our own process; potash from kainit. Found: Phosphoric acid, soluble, 2.98 ; reverted, 4.85 ; insoluble, 2.66 ; total, I0.49; available, 7.83 ; nitrogen, I.05; potash, 2. I I availability of nitrogen, 88 ; chlorine, I.3.

\section{DETRICK'S IMPERIAL COMPOUND. W. S.} Corrothers, Agent, Little Falls, W. Va. Guarantee: (As above.) Found: Phosphoric acid, soluble, 4.86; reverted, 3.36 ; insoluble, I.09; total, 9.3I ; available, 8.22; nitrogen, 0.92 ; potash, 2.I5; availability of nitrogen, 67 ; chlorine, 3.6.

Remark: Chlorine excessive.

5598. DETRICK'S IMPERIAL COMPOUND. A. P. Russell \& Co., Agent, Buckhannon, W. Va. Guarantee: (As above.) Found: Phosphoric acid, soluble, 6.09; reverted, 2.28; insoluble, o.99; total, 9.36 ; available, 8.37 ; nitrogen, r.03; potash, 2.36 ; availability of nitrogen, $8 \mathrm{I}$; chlorine, 2.7 .

\section{DETRICK'S CORN AND OATS FERTILIZER.} A. P. Russell \& Co., Agent, Buckhannon, W. V. Guarantee: Phosphoic acid, soluble, 7 ; reverted, 2 ; insoluble, I; total, Io; available, 9 ; nitrogen, .82; potash, 3 ; phosphoric acid from dissolved animal bone and dissolved phosphate rock; nitrogen not less than one-tenth from bone tankage or not less than one-tenth from fish, the remainder from base goods, made from animal matter, treated by our own process; potash, $1 / 4$ to $3 / 4$ from manure salt, $1 / 4$ to $3 / 4$ from kainit. Found: Phosphoric acid, 
soluble, 5.97 ; reverted, 3.12 ; insoluble, 0.95 ; total, 10.04; available, 9.09; nitrogen, .93; potash, 2.90 ; availability of nitrogen, 7 I ; chlorine, 2.I.

56I8. DETRICK'S CORN AND OATS FERTILIZER. W. S. Corrothers, Agent, Little Falls. Guarantee: (As above.) Found: Phosphoric acid, soluble, 7.18; reverted, 2.83; insoluble, 0.67 ; total, Io.68; available, I0.01 ; nitrogen, 0.97 ; potash, 3.53 ; availability of nitrogen, 82 ; chlorine, I.6.

5597. DETRICK'S STANDARD POTASH FERTILIZER. A. P. Russell \& Co., Agent, Buckhannon, W. Va. Guarantee: Phosphoric acid, soluble, 4; reverted, 2 ; insoluble, I; total, 7 ; available, 6; nitrogen, I.24; potash, 5 ; phosphoric acid from dissolved animal bone and dissolved phosphate rock; nitrogen not less than one-tenth from bone tankage, not less than one-tenth from fish, the remainder from base goods, made from animal matter, treated by our own process; potash $I / 4$ to $3 / 4$ from manure salt and $1 / 4$ to $3 / 4$ from kainit. Found: Phosphoric acid, soluble, 2.5I ; reverted, 3.66; insoluble, I.25; total, 7.42; available, 6.17 ; nitrogen, I.22; potash, 5.80 ; availability of nitrogen, 78 ; chlorine, 2.2 .

5489. GREAT EASTERN CORN FERTILIZER. J. D. Anderson, Agent, Ronceverte,.W. Va. Guarantee: Phosphoric acid, soluble, 6; reverted, 2 ; insoluble, I ; total, 9; available, 8 ; nitrogen, 0.82 ; potash, 4 ; phosproric acid from dissolved animal bone and dissolved phosphate rock; nitrogen, not less than onetenth from bone tankage, or not less than one-tenth from fish, the remainder from base goods, made from animal matter, treated by our own process; potash, $1 / 4$ to $3 / 4$ from manure salts, $1 / 4$ to $3 / 4$ from kainit. Found: Phosphoric acid, soluble, 4.78 ; reverted, 3.40 ; insoluble, 0.93 ; total, 9.I I ; available, 8.18; nitrogen, 95 ; potash, 4.01 ; availability of nitrogen, 79 ; chlorine, 2.6.

5536. GREAT EASTERN CORN FERTILIZER. Draper England, Agent, Belington, W. Va. Guarantee: (As above.) Found: Phosphoric acid, soluble, 5.12; reverted, 2.63; insolu- 
ble, I.22 ; total, 8.97 ; available, 7.75 ; nitrogen, 0.86 ; potash, 3.97 ; availability of nitrogen, 74 ; chlorine, I.8.

5491. GREAT EASTERN DISSOLVED BONE. J. D. Anderson, Agent, Ronceverte, W. Va. Guarantee: Phosphoric acid, soluble, I2 ; reverted, 2 ; insoluble, I ; total, I5; available, I4; phosphoric acid from dissolved phosphate rock. Found: Phosphoric acid, soluble, I0.4I ; reverted, 4.4I ; insoluble, 0.58 ; total, I 5.40 ; available, I4.82.

5537. GREAT EASTERN DISOLVED BONE. Draper England, Agent, Belington, W. Va. Guarantee: (As above.) Found: Phosphoric acid, soluble, II.I7; reverted, 4.20 ; insoluble, $0.5 \mathrm{I}$; total, I 5.88 ; available, I 5.37 .

5724. GREAT EASTERN DISSOLVED BONE. J. D. Anderson, Agent, Ronceverte, W. Va. Guarantee: (As above.) Found: Phosphoric acid, soluble, Io.68; reverted, 4.40 ; insoluble, 0.54 ; total, I 5.62 ; available, I 5.08 .

5746. GREAT EASTERN DISSOLVED BONE. A. G. Chrislip, Agent, Philippi, W. Va. Guarantee: (As above.) Found: Phosphoric acid, soluble, 9.79; reverted, 4.67; insoluble, I.Io; total, I5.56; available, I4.46.

5766. GREAT EASTERN DISSOLVED BONE. C. E. Casler, Agent, Berkeley Springs, W. Va. Guarantee: (As above.) Found: Phosphoric acid, soluble, I2.22; reverted, 3.45; insoluble, 0.27 ; total, I 5.94; available, I5.67.

5492. GREAT EASTERN VEGETABLE, VINE AND TOBACCO. J. D. Anderson, Agent, Ronceverte, W. Va. Guarantee: Phosphoric acid, soluble, 6; reverted, 2 ; insoluble, I ; total, 9; available, 8; nitrogen, 2.06; potash, 3; phosphoric acid from dissolved animal bone and dissolved phosphate rock; nitrogen, not less than one-tenth from bone tankage, not less than one-tenth from fish, the remainder from base goods, made from animal matter, treated by our own process; potash $1 / 4$ to $3 / 4$ 
from manure salt, and $1 / 4$ to $3 / 4$ from kainit. Found: Phosphoric acid, soluble, 4.63 ; reverted, 3.57 ; insoluble, I.60; total, 9.80 ; available, 8.20 ; nitrogen, 1.76 ; potash, 2.98 ; availability of nitrogen, $8 \mathrm{I}$; chlorine, 2.4 .

Remark: Nitrogen low.

55I7. GREAT EASTERN VEGETABLE, VINE AND TOBACCO. T. M. Harbor, Agent, Hurricane, W. Va. Guarantec: (As above.) Found: Phosphoric acid, soluble, 5.62; reverted, 4.02 ; insoluble, I.66; total, II ; available, 9.34 ; nitrogen, 2.3I ; potash, 2.96; availability of nitrogen, 85 ; chlorine, 0.7 .

55I6. GREAT EASTERN ENGLISH WHEAT GROWER. T. M. Harbor, Agent, Hurricane, W. Va. Guarantee: Phosphoric acid, soluble, 6; reverted, 2; insoluble, I; total, 9; available, 8 ; nitrogen, .82; potash, 2 ; phosphoric acid from dissolved animal bone and dissolved phosphate rock; nitrogen, not less than one-tenth from bone tankage, or not less than one-tenth from fish, the remainder from base goods, made from animal matter, treated by our own process; potash, from kainit. Found: Phosphoric acid, soluble, 4.22; reverted, 4.56 ; insoluble, 2.53 ; total, II.2I ; available, 8.69; nitrogen, .I.I5; potash, 2 ; availability of nitrogen, 90 ; chlorine, I.5.

5722. GREAT EASTERN ENGLISH WHEAT GROWER. J. D. Anderson, Agent, Ronceverte, W. Va. Guarantee: (As above.) Found: Phosphoric acid, soluble, 3.84; reverted, 4.II ; insoluble, I.80; total, 9.75 ; available, 7.95 ; nitrogen, I.05; potash, 2.08; availability of nitrogen, 87 ; chlorine, 3.4 .

Remark: Chlorine excessive.

5745. GREAT EASTERN ENGLISH WHEAT GROWER. A. G. Chrislip, Agent, Philippi, W. Va. Guarantee: (As above.) Found: Phosphoric acid, soluble, 4.80; reverted, 3.I4; insoluble, 0.82 ; total, 8.76 ; available, 7.94 ; nitrogen, 0.88 ; potash, 2.24 ; availability of nitrogen, 72 ; chlorine, 3.5 .

Remark: Chlorine excessive. 
5535. GREAT EASTERN SOLUBLE BONE AND POTASH. Draper England, Agent, Belington, W. Va. Guarantee: Phosphoric acid, soluble, 8; reverted, 2; insoluble, I ; total, I I ; available, Io; potash, 2 ; phosphoric acid from dissolved phosphate rock; potash from kainit. Found: Phosphoric acid, soluble, 6.72 ; reverted, 2.92 ; insoluble, 0.84 ; total, I0.48; available, 9.64; potash, 2.26 ; chlorine, 5.7 .

Remark: Chlorine very high.

5764. GREAT EASTERN SOLUBLE BONE AND POTASH. C. E. Casler, Agent, Berkeley Springs, W. Va. Guarantee: (As above.) Found: Phosphoric acid, soluble, 5.05 ; reverted, 5.13 ; insoluble, .58; total, IO.76; available, IO.I8; potash, 2.00 ; chlorine, 3.9 .

Remark: Chlorine excessive.

5538. GREAT EASTERN UNAMMONIATED WHEAT $S P E C I A L$. Draper England, Agent, Belington, W. Va. Guarantee: Phosphoric acid, soluble, Io; reverted, 2 ; insoluble, I; total, I3; available, I2 ; phosphoric acid from dissolved phosphate rock. Found: Phosphoric acid, soluble, 8.34; reverted, 5.24; insoluble, 0.65 ; total, I4.23; available, I 3.58 .

572I. GREAT EASTERN HIGH GRADE BONE AND POTASH. J. D. Anderson, Agent, Ronceverte, W. Va. Guarantee: Phosphoric acid, soluble, 8; reverted, 2; insoluble, I ; total, I I ; available, Io ; potash, 5 ; phosphoric acid from dissolved phosphate rock; potash $1 / 4$ to $3 / 4$ from muriate of potash, $1 / 4$ to $3 / 4$ from manure salts. Found: Phosphoric acid, soluble, 6.or; reverted, 4.04; insoluble, I.02; total, II.07; available, I0.05; potash, 5.23 ; chlorine, 2.I.

5747. GREAT EASTERN HIGH GRADE BONE AND POTASH. A. G. Chrislip, Agent, Philippi, W. Va. Guarantee: (As above.) Found: Phosphoric acid, soluble, 6.oI ; reverted, 3.50 ; insoluble, 0.83 ; total, I0.34; available, $9.5 \mathrm{I}$; potash, 4.56; chlorine, 2.7 . 
Remark: Chlorine excessive. Available phosphoric acid low. Potash low.

5765. GREAT EASTERN JAPANESE WHEAT GROWER. C. E. Casler, Agent, Berkeley Springs, W. Va. Guarantee: Phosphoric acid, soluble, 4; reverted, 2 ; insoluble, I; total, 7 ; available, 6; potash, 3 ; phosphoric acid from dissolved phosphate rock; potash from kainit. Found: Phosphoric acid, soluble, 2.56; reverted, 4.03; insoluble, 0.70; total, 7.29; available, 6.59; potash, 3.25 ; chlorine, 3.7 .

Remark: Chlorine excessive.

5525. LAZARETTO HIGH GRADE DISSOLVED BONE AND POTASH. A. G. Chrislip, Agent, Philippi, W. Va. Guarantee: Phosphoric acid, soluble, IO; reverted, 2 ; insoluble, I; total, I3; available, I2; potash, 5 ; phosphoric acid from dissolved phosphate rock; potash from muriate of potash. Found: Phosphoric acid, soluble, 8.83 ; reverted, 2.67 ; insoluble, 0.87 ; total, I2.37; available, II.50; potash, 5.26; chlorine, 2.I.

Remark: Source of potash equivalent to kainit.

5744. LAZARETTO HIGH GRADE DISSOLVED BONE $A N D$ POTASH. A. G. Chrislip, Agent, Philippi, W. Va. Guarantee: (As above.) Found: Phosphoric acid, soluble, 8.83; reverted, 2.88; insoluble, 0.90 ; total, I2.6I ; available, II.7I ; potash, 5.08; chlorine, 2.I.

Remark: Phosphoric acid low; source of potash equivalent to kainit.

5582. LAZARETTO CROP GROWER. T. B. Drummond \& Co., Agent, Buckhannon, W. Va. Found: Phosphoric acid, soluble, 4.68; reverted, 3.30 ; insoluble, I.4I ; total, 9.39; available, 7.98 ; nitrogen, I.52; potash, 2.45 ; availability of nitrogen, 84 ; chlorine, 2.4 .

5583. LAZARETTO BONE COMPOUND. T. B. Drummond \& CO., Agent, Buckhannon, W. Va. Guarantee: Phosphoric acid, soluble, 7 ; reverted, 2 ; insoluble, I ; total, Io; avail- 
able, 9; nitrogen, I.03; potash, 3; phosphoric acid from dissolved animal bone and dissolved phosphate rock; nitrogen not less than one-tenth from bone tankage, or not less than one-tenth from fish, the remainder from base goods, made from animal matter, treated by our own process; potash $1 / 4$ to $3 / 4$ from manure salts; $1 / 4$ to $3 / 4$ from kainit. Found: Phosphoric acid, soluble, 4.99; reverted, 3.78; insoluble, I.I6; total, 9.83; available, 8.67; nitrogen, I.34; potash, 2.85 ; availability of nitrogen, 77 ; chlorine, I.9.

Remark: Available phosphoric acid low.

5784. LAZARETTO BONE COMPOUND. T. B. Drummond \& Co., Agent, Buckhannon, W. Va. Guarantee: (As above.) Found: Phosphoric acid, soluble, 8.12 ; reverted, 2.29; insoluble, .92 ; total, II.33; available, IO.4I ; nitrogen, .98; potash, 3 ; availability of nitrogen, 84 ; chlorine, 2.5 .

5584. LAZARETTO DISSOLVED BONE PHOSPHATE. T. B. Drummond \& Co., Agent, Buckhannon, W. Va. Guarantee: Phosphoric acid, soluble, I2; reverted, 2 ; insoluble, I ; total, I5; available, I4; phosphoric acid from dissolved phosphate rock. Found: Phosphoric acid, soluble, I2.09; reverted, $3: 67$; insoluble, o.I8; total, I 5.94; available, I 5.76.

5596. LAZARETTO SPECIAL POTATO AND TOBACCO FERTILIZER. T. B. Drummond \& Co., Agent, Buckhannon, W. Va. Guarantee: Phosphoric acid, soluble, 6; reverted, 2 ; insoluble, I ; total, 9; available, 8; nitrogen, .82; potash, 4; phosphoric acid from dissolved animal bone and dissolved phosphate rock; potash $1 / 4$ to $3 / 4$ from manure salt, $1 / 4$ to $3 / 4$ from kainit; nitrogen, not less than one-tenth from bone tankage, or not less than one-tenth from fish, the remainder from base goods, made from animal matter, treated by our own process. Found: Phosphoric acid, soluble, 3.9I ; reverted, 3.09; insoluble, I.38; total, 8.38 ; available, 7.00 ; nitrogen, 0.89 ; potash, 4.I I ; availability of nitrogen, 75 ; chlorine, 2.I.

Remark: Available phosphoric acid low. 
5458. MARYLAND AMMONIATEDD BONE. J. L. Woodyard, Pruntytown, W. Va. Guarantee: Phosphoric acid, soluble, 6 ; reverted, 2 ; insoluble, I ; total, 9; available, 8 ; nitrogen, 1.65 ; potash, 3 ; phosphoric acid from dissolved animal bone and dissolved phosphate rock; nitrogen, not less than one-tenth from bone tankage; not less than one-tenth from fish, the remainder from base goods, made from aninal matter treated by our own process; potash $I / 4$ to $3 / 4$ from manure salt, $1 / 4$ to $3 / 4$ from kainit. Found: Phosphoric acid, soluble, 4.I2; reverted, 5.35; insoluble, I.7I ; total, I I.I8; available, 9.47 ; nitrogen, I.68; potash, 3.26 ; availability of nitrogen, 86 ; chlorine, 1.3.

5459. MARYLAND BONO-SUPERPHOSPHATE. J. L. Woodyard, Agent, Pruntytown, W. Va. Guarantee: Phosphoric acid, soluble, 8 ; reverted, 2 ; insoluble, I ; total, II ; available, Io ; potash, 2; phosphoric acid from dissolvd phosphate rock; potash from kainit. Found: Phosphoric acid, soluble, 5.IO; reverted, 5.81 ; insoluble, o.86; total, II.77; available, I0.91 ; potash, 2.62 ; chlorine, 1.9.

5565. MARY LAND DISSOLVED S. C. BONE. W. S. Stout, Agent, Harrisville, W. Va. Guarantee: Phosphoric acid, soluble, I2; reverted, 2 ; insoluble, I; total, I5; available, I4; phosphoric acid from dissolved phosphate rock. Fonnd: Phosphoric acid, soluble, 9.38 ; reverted, 5.38 ; insoluble, I.45; total, I6.21; available, I4.76.

5486. PACIFIC DISSOLVED BONE AND POTASH. H. E. Sharp, Agent, Alderson, W. Va. Guarantee: Phosphoric acid, soluble, 8 ; reverted, 2 ; insoluble, I ; total, II ; available, Io ; potash, 2; phosphoric acid from dissolved phosphate rock; potash from kainit. Found: Phosphoric acid, soluble, 6.5x; reverted, 3.03; insoluble, I.I5; total, I0.69; available, 9.54; potash, 2.19; chlorine, 3 .

Remark: Available phosphoric acid low.

5552. SUSQUEHANNA XXV. PHOSPHATE. J. H. Miller \& Son, Agent, Martinsburg, W. Va. Guarantee: Phos- 
phoric acid, soluble, 6; reverted, 2 ; insoluble, I ; total, 9 ; available, 8 ; nitrogen, 0.82 ; potash, I ; phosphoric acid from dissolved phosphate rock and dissolved animal bone, nitrogen, not less than one-tenth from bone tankage, or not less than one-tenth from fish, the remainder from base goods, made from animal matter, treated by our own process; potash, from kainit. Found: Phosphoric acid, soluble, 3.80 ; reverted, 4.05; insoluble, I.39; total, 9.24 ; available, 7.85 ; nitrogen, 0.84 ; potash, I.27; availability of nitrogen, 72 ; chlorine, 2.9 .

\section{SUSQUEHANNA POTATO AND TOBACCO} PHOSPHATE. J. H. Miller \& Son, Agent, Martinsburg, W. Va. Found: Phosphoric acid, soluble, 4.36; reverted, 4.06; insoluble, I.28; total, 9.70; available, 8.42 ; nitrogen, I.6I ; potash, 8.oI ; availability of nitrogen, 83 ; chlorine, I.2.

5457. WILLIAMS' \& CLARK'S DISSOLVED'BONE AND POTASH. W. A. Aldridge, Agent, Seebert, W. Va. Guarantee: Phosphoric acid, soluble, 8; reverted, 2 ; insoluble, I ; total, II ; available, Io ; potash, 2 ; phosphoric acid from dissolved phosphate rock; potash from kainit. Found: Phosphoric acid, soluble, 6.95; reverted, 3.I6; insoluble, I.02; total, II.I3; available, IO.II ; potash, 2.20 ; chlorine, I.

5524. WILLIAM'S \& CLARK'S DISSOLVED BONE AND POTASH. A. G. Chrislip, Agent, Philippi, W. Va. Guarantee: (As above. Found: Phosphoric acid, soluble, 5.76 ; reverted, 2.34 ; insoluble, 2.54 ; total, Io.64; available, 8.Io; potash, 2.47 ; chlorine, 4.7 .

Remark: Chlorine excessive.

5649. WILLIAMS' \& CLARK'S DISSOLVED BONE AND POTASH. Orie Carmen, Agent, Wellsburg, W. Va. Guarantee: (As above.) Found: Phosphoric acid, soluble, 4.22 ; reverted, 5.49; insoluble, I.94; total, I I.65; available, 9.7I ; potash, 2.I6; chlorine, I.5.

5494. WILLIAMS' \& CLARK'S GOOD GROWER PO- 
TATO PHOSPHATE. Mossman Bros., Agent, Huntington, W. Va. Guarantee: Phosphoric acid, soluble, 4 ; reverted, 2 ; insoluble, I ; total, 7 ; available, 6 ; nitrogen, I.24; potash, 5 ; phosphoric acid from dissolved animal bone and dissolved phosphate rock; nitrogen, not less than one-tenth from bone tankage, not less than one-tenth from fish, the remainder from base goods, made from animal matter, treated by our own process; potash $1 / 4$ to $3 / 4$ from manure salt, $1 / 4$ to $3 / 4$ from kainit. Found: Phosphoric acid, soluble, 2.7 I reverted, 4.50 ; insoluble, 1.80 ; total, 9.01 ; available, 7.21 ; nitrogen, I.48; potash, 5.55 ; availability of nitrogen, 76 ; chlorine, $\mathrm{I}$.

5530. WILLIAMS' \& CLARK'S GOOD GROWER POTATO PHOSPHATE. A. G. Chrislip, Agent, Philippi, W. Va. Guarantee: (As above.) Found: Phosphoric acid, soluble, 3.28; reverted, 3.oI ; insoluble, I.28; total, 7.57 ; available, 5.29; nitrogen, I.I7; potash, 4.75 ; availability of nitrogen, 78 ; chlorine, 2.2 .

Remark: Available phosphoric acid low.

5626. WILLIAMS' \& CLARK'S GOOD GROWER POTATO PHOSPHATE. Curry \& Rodgers, Agent, Marlinton, W. Va. Guarantee: (As above.) Found: Phosphoric acid, soluble, 2.37 ; reverted, 4.25 ; insoluble, .92 ; total, 7.54 ; available, 6.62 ; nitrogen, I.23; potash, 3.98 ; availability of nitrogen, 87; chlorine, 2.9 .

5696. WILLIAMS' \& CLARK'S GOOD GROWER POTATO PHOSPHATE. Belington Ice \& Bottling Co., Belington, W. Va. Guarantee: (As above. Found: Phosphoric acid, soluble, 2.49; reverted, 3.38; insoluble, 1.29; total, 7.16; available, 5.87 ; nitrogen, I.I8; potash, 4.79 ; availability of nitrogen, 79 ; chlorine, 2.7 .

5523. WILLIAMS' \& CLARK'S ACORN ACID PHOSPHATE. A. G. Chrislip, Agent, Philippi, W. Va. Guarantee: Phosphoric acid, soluble, I2; reverted, 2 ; insoluble, I ; total, I5; available, I4; phosphoric acid from dissolved phosphate rock. 
Found: Phosphoric acid, soluble, Ir.04; reverted, 3.I6; insoluble, 0.54 ; total, I4.74; available, I4.20.

5694. WILLIAMS' \& CLARK'S ACORN ACID PHOSPHATE. Belington Ice \& Bottling Co., Agent, Belington, W. Va. Guarantee: (As above.) Found: Phosphoric acid, soluble, I2.4I ; reverted, 3.72 ; insoluble, 0.32 ; total, I6.45; available, I6.I3.

5742. WILLIAMS' \& CLARK'S ACORN ACID PHOSPHATE. A. G. Chrislip, Agent, Philippi, W. Va. Guarantee: (As above.) Found: Phosphoric acid, soluble, I2.I4; reverted, 3.66; insoluble, o.33; total, I6.13; available, I5.80.

5534. WILLIAMS' \& CLARK'S ROYAL BONE PHOSPHATE. Curry \& Rogers, Agent, Marlinton, W. Va. Guarantee: Phosphoric acid, soluble, 6; reverted, 2; insoluble, I; total, 9; available, 8; nitrogen, I.03; potash, 2; phosphoric acid from dissolved animal bone and dissolved phosphate rock; nitrogen, not less than one-tenth from tankage, not less than one-tenth from fish, the remainder from base goods, made from animal matter, treated by our own process; potash, from kainit. Found: Phosphoric acid, soluble, 4.86; reverted, 3.4I ; insoluble, I.I3; total, 9.40 ; available, 8.27 ; nitrogen, 0.99 ; potash, 2.64; availability of nitrogen, 79; chlorine, 2.4 .

5562. WILLIAMS' \& CLARK'S ROYAL BONE PHOSPHATE. O. R. Carmen, Agent, Wellsburg, W. Va. Guarantee: (As above.) Found: Phosphoric acid, soluble, 5.86; reverted, 2.25 ; insoluble, I.23; total, 9.34; available, 8.II ; nitrogen, 0.98 ; potash, 2.25; availability of nitrogen, 76 ; chlorine, r.4. 5651. WILLIAMS' \& CLARK'S ROYAL BONE PHOS-

PHATE. Orie Carmen, Agent, Wellsburg, W. Va. Guarantee: (As above.) Found: Phosphoric acid, soluble, 4.4I ; reverted, $3.8 \mathrm{I}$; insoluble, I.84; total, I0.06; available, 8.22 ; nitrogen, 9I ; potash, 2.50; availability of nitrogen, 84 ; chlorine, r.6.

Remark: Nitrogen low. 
5665. WILLIAMS' \& CLARK'S ROYAL BONE PHOSPHATE. W. R. Dudley, Agent, Wheeling, W. Va. Guarantee: (As above.) Found: Phosphoric acid, soluble, 4.22;. reverted, 4.23 ; insoluble, I.98; total, 10.43; available, 8.45 ; nitrogen, 0.84 ; potash, 2.20 ; availability of nitrogen, $8 \mathrm{I}$; chlorine; I.7.

Remark: Nitrogen low.

5692. WILLIAMS' \& CLARK'S ROYAL BONE PHOSPHATE. Belington Ice \& Bottling Co., Belington, W. Va. Guarantee: (As above.- Found: Phosphoric acid, soluble, 4.I6; reverted, 4.I0; insoluble, 2.12 ; total, I0.38; available, 8.26; nitrogen, I.II ; potash, 224; availability of nitrogen, 0.88 ; chlorine, 3.6.

Remark: Chlorine excessive.

5666. WILLIAMS' \& CLARK'S PROLIFIC CROP PRODUCER. W. R. Dudley, Agent, Wheeling, W. Va. Guarantee: Phosphoric acid, soluble, 5; reverted, 2; insoluble, I; total, 8; available, 7 ; nitrogen, 0.82 ; potash, $\mathrm{I}$; phosphoric acid from dissolved animal bone and dissolved phosphate rock; nitrogen, not less than one-tenth from bone tankage, or not less than one-tenth from fish, the remainder from base goods, made from animal matter, treated by our own process; potash from kainit. Found: Phosphoric acid, soluble, 3.7I; reverted, 3.65 ; insoluble, I.7I; total, 9.07 ; available, 7.36 ; nitrogen, 0.97 ; potash, I.3I ; availability of-nitrogen, 83 ; chlorine, I.7.

5693. WILLIAMS' \& CLARK'S PROLIFIC CROP PRODUCER. Belington Ice \& Bottling Co., Belington, W. Va. Guarantee: (As above.) Found: Phosphoric acid, soluble, 6.78; reverted, 0.50; insoluble, 0.95 ; total, 8.23 ; available, 7.28 ; nitrogen, 0.89 ; potash, I.5I ; availability of nitrogen, 78 ; chlorine, 6.I.

Remark: Chlorine excessive.

5743. WILLIAMS' \& CLARK'S PROLIFIC CROP PRODUCER. A. G. Chrislip, Agent, Philippi, W. Va. Guarantee: (As above.) Found: Phosphoric acid, soluble, 4.54; reverted, 
2.80; insoluble, I ; total, 8.34; available, 7.34; nitrogen, 0.93 ; potash, I.63; availability of nitrogen, 72 ; chlorine, 4.9 .

Remark: Chlorine excessive.

5490. WHEELER'S POTATO MANURE. J. D. Anderson, Agent, Ronceverte, W. Va. Guarantee: Phosphoric acid, soluble, 6; reverted, 2 ; insoluble, I ; total, 9 ; available, 8 ; nitrogen, 2.06; potash, 3 ; phosphoric acid from dissolved animal bone and dissolved phosphate rock; nitrogen, not less than one-tenth from bone tankage, not less than one-tenth from fish, the remainder from base goods, made from animal matter, treated by our own process; potash, $I / 4$ to $3 / 4$ from manure salt, $1 / 4$ to $3 / 4$ from kainit. Found: Phosphoric acid, soluble, 4.40; reverted, 3.80 ; insoluble, I.64; total, 9.84 ; available, 8.20 ; nitrogen, I.90; potash, 2.95 ; availability of nitrogen, 86 ; chlorine, 2.2.

Remark: Nitrogen low.

5720. WHEELER'S ROYAL WHEAT GROWER. J. D. Anderson, Agent, Ronceverte, W. Va. Guarantee: Phosphoric acid, soluble, 6; reverted, 2 ; insoluble, I ; total, 9; available, 8 ; nitrogen, .82; potash, 2 ; phosphoric acid from dissolved animal bone and dissolved phosphate rock, nitrogen, not less than onetenth from bone tankage, or not less than one-tenth from fish, the remainder from base goods, made from animal matter, treated by our own process; potash from kainit. Found: Phosphoric acid, soluble, 4.6I ; reverted, 3.44 ; insoluble, I.97; total, I0.02; available, 8.05 ; nitrogen, 0.95 ; potash, I.89; availability of nitrogen, 85 ; chlorine, 4 .

Remark: Chlorine excessive.

5690. WHEELER'S ROYAL WHEAT GROWER. Thomas Nuzum, Agent, Grafton, W. Va. Guarantee: (As above.) Found: Phosphoric acid, soluble, 3.or ; reverted, 4.59; insoluble, I.o9; total, 8.69; available, 7.60; nitrogen, 0.79 ; potash, $2.8 \mathrm{I}$; availability of nitrogen, 84 ; chlorine, 4.8 .

Remark: Chlorine excessive. Phosphoric acid low.

5723. WHEELER'S WHEAT AND CLOVER FERTIL- 
IZER. J. D. Anderson, Agent, Ronceverte, W. Va. Guarantee: Phosphoric acid, soluble, 8 ; reverted, 2 ; insoluble, I ; total, I I.00; available, I0.00 ; potash, 2 ; phosphoric acid from dissolved phosphate rock; potash from kainit. Found: Phosphoric acid, soluble, 6.40; reverted, 2.52 ; insoluble, .77; total, Io.69; available, 9.92 ; potash, 2.10 ; chlorine, 3 .

5767. WHEELER'S WHEAT AND CLOVER FERTIL$I Z E R$. C. E. Casler, Agent, Berkeley Springs, W. Va. Guars antee: (As above.) Found: Phosphoric acid, soluble, 6.46; reverted, 3.50 ; insoluble, .22 ; total, Io.I I ; available, 9.89 ; potash, 2.18; chlorine, 2.9.

5763. WHEELER'S ELECTRICAL DISSOLVED BONE. C. E. Casler, Agent, Berkeley Springs, W. Va. Guarantee: Phosphoric acid, soluble, I2 ; reverted, 2; insoluble, I; total, I5; available, I4; phosphoric acid from dissolved phosphate rock. Found: Phosphoric acid, soluble, II.26; reverted, 3.53 ; insoluble, I.07; total, I5.86; available, I4.79.

5465. ZELL'S LITTLEE GIANT. Benson Jacobs, Agent, Little Falls, W. Va. Guarantee: Phosphoric acid, soluble, 5; reverted, 2 ; insoluble, I ; total, 8 ; available, 7 ; nitrogen, 0.82 ; potash, I ; phosphoric acid from dissolved animal bone and dissolved phosphate rock; nitrogen, not less than one-tenth from bone tankage, or not less than one-tenth from fish, the remainder from base goods, made from animal matter, treated by our own process; potash from kainit. Found: Phosphoric acid, soluble, 5.62; reverted, 2.50; insoluble, I.oI ; total, 9.I3; available, 8.I2; nitrogen, 1.04; potash, I.22; availability of nitrogen, 75 ; chlorine, 2.2 .

5493. ZELL'S LITTLE GIANT. Mossman Bros., Agent, Huntington, W. Va. Guarantee: (As above.) Found: Phosphoric acid, soluble, 2.38; reverted, 5.39; insoluble, 2.62; total, I0.39; available, 7.77 ; nitrogen, 0.99 ; potash, I.24; availability of nitrogen, 75 ; chlorine, I.I. 
5533. ZELL'S LITTLE GIANT. Curry \& Rogers, Marlinton, W. Va. Guarantee: (As above.) Found: Phosphoric acid, soluble, 4.70; reverted, 2.93; insoluble, I.27; tota1, 8.90; available, 7.63 ; nitrogen, 0.94 ; potash, I.I 3 ; availability of nitrogen, 77 ; chlorine, 2.5 .

5622. ZELL'S LITTLE GIANT. Cicero Phillips, Agent, Belington, W. Va. Guarantee: (As above.) Found: Phosphoric acid, soluble, 4.58 ; reverted, 2.78; insoluble, .84; total, 8.20 ; available, 7.36 ; nitrogen, 0.99 ; potash, I.33; availability of nitrogen, 72 ; chlorine, 3.2 .

Remark: Chlorine excessive.

5636. ZELL'S LITTLE GIANT. Benson Jacobs, Agent, Little Falls, W. Va. Guarantee: (As above.) Found: Phosphoric acid, soluble, 3.68; reverted, 3.I9; insoluble, .97; total, 7.84 ; available, 6.87 ; nitrogen, 0.87 ; potash, 1.29; availability of nitrogen, 9 I ; chlorine, $5 \cdot 5$.

Remark: Chlorine excessive.

5643. ZELL'S LITTLE GIANT. C. R. Carmen, Agent, Wellsburg, W. Va. Guarantee: (As above.) Found: Phosphoric acid, soluble, 5.I8; reverted, 3.II ; insoluble, I.4I ; total, 9.70; available, 8.29; nitrogen, I.05; potash, 2.63 ; availability of nitrogen, 82 ; chlorine, I.2.

5687. ZELL'S LITTLE GIANT. Parley DeBerry, Agent, Terra Alta, W. Va. Guarantee: (As above.) Found: Phosphoric acid, soluble, 5.08; reverted, 3.37 ; insoluble, I.23; total, 9.68; available, 8.45 ; nitrogen, .97 ; potash, I.75; availability of nitrogen, 77 ; chlorine, 2.4 .

5770. ZELL'S LITTLE GIANT. R. Hunter, Agent, Berkeley Springs, W. Va. Guarantee: (As above.) Found: Phosphoric acid, soluble, 4.09; reverted, 3.03; insoluble, I.09; total, 8.2I ; available, 7.I2; nitrogen, .93; potash, I.49; availability of nitrogen, 7I ; chlorine, 5.3 .

Remark: Chlorine excessive. 
5466. ZELL'S ECONOMIZER PHOSPHATE. Benson Jacobs, Agent, Little Falls, W. Va. Guarantee: Phosphoric acid, soluble, 6 ; reverted, 2 ; insoluble, I ; total, 9; available, 8; nitrogen, .82 ; potash, 2 ; phosphoric acid from dissolved animal bone and dissolved phosphate rock; nitrogen, not less than onetenth from bone tankage, or not less than one-tenth from fish, the remainder from base goods, made from animal matter, treated by our own process; potash from kainit. Found: Phosphoric acid, soluble, 6.77; reverted, 2.38; insoluble, r.23; total, 10.38; available, 9.15; nitrogen, 0.86 ; potash, 2.27 ; availability of nitrogen, 79; chlorine, 2 .

5488. ZELL'S ECONOMIZER PHOSPHATE. Johnson \& Gwinn, Agent, Alderson, W. Va. Guarantee: (As above.) Found: Phosphoric acid, soluble, 6.44; reverted, 2.33; insoluble, I.or ; total, 9.80; available, 8.79; nitrogen, r.o6; potash, 2.85; availability of nitrogen, 85 ; chlorine, 2.5 .

5640. ZELL'S ECONOMIZER PHOSPHATE. C. R. Carmen, Agent, Wellsburg, W. Va. Guarantee: (As above.) Found: Phosphoric acid, soluble, 5.40; reverted, 2.62 ; insoluble, 0.63 ; total, 8.65 ; available, 8.02 ; nitrogen, 1.06 ; potash, 2.30; availability of nitrogen, 87 ; chlorine, r.8.

5593. ZELL'S ECONOMIZER PHOSPHATE. Exchange Mill Co., Agent, Grafton, W. Va. Guarantee: (As above.) Found: Phosphoric acid, soluble, 3.68; reverted, 4.24; insoluble, r.33; total, 9.25 ; available, 7.92 ; nitrogen, 1.03; potash, 2.27; availability of nitrogen, 73 ; chlorine, 2.4 .

5728. ZELL'S ECONOMIZER PHOSPHATE. Johnson \& Gwinn, Agent, Alderson, W. Va. Guarantee: (As above.) Found: Phosphoric acid, soluble, 5.37; reverted, 3.or ; insoluble, I.I 5 ; total, 9.53 ; available, 8.38 ; nitrogen, .9I; potash, 2.44; availability of nitrogen, 70 ; chlorine, 3 .

5467. ZELL'S DISSOLVED BONE PHOSPHATE. Benson Jacobs, Agent, Little Falls, W. Va. Guarantee: Phosphoric 
acid, soluble, I2 ; reverted, 2 ; insoluble, I ; total, I 5 ; available, I4; phosphoric acid from dissolved phosphate rock. Found: Phosphoric acid, soluble, Io; reverted, 5.8I ; insoluble, 0.73 ; total, I6.64; available, I 5.8I.

5487. . ZELL'S DISSOLVED BONE PHOSPHATE. Johnson \& Gwinn, Agent, Alderson, W. Va. Guarantee: (As above.) Found: Phosphoric acid, soluble, II.I9; reverted, 4.48 ; insoluble, 0.78 ; total, I6.35; available, I 5.57 .

55I9. ZELL'S DISSOLVED BONE PHOSPHATE. Philippi Mill Co., Philippi, W. Va. Guarantee: (As above.) Found: Phosphoric acid, soluble, ro.82; reverted, 4.69; insoluble, .58; total, I6.o9; available, I 5.5I.

5603. ZELL'S DISSOLVED BONE PHOSPHATE. Exchange Mill Co., Agent, Grafton, W. Va. Guarantee: (As above.) Found: Phosphoric acid, soluble, I0.30; reverted, 4.29 ; insoluble, o.68; total, I 5.27; available, I4.59.

5632. ZELL'S DISSOLVED BQNE PHOSPHATE. Benson Jacobs, Agent, Little Falls, W. Va. Guarantee: (As above.) Found: Phosphoric acid, soluble, Io.87; reverted, 4.76; insoluble, o.I 3 ; total, I 5.76 ; available, I 5.63 .

5729. ZELL'S DISSOLVED BONE PHOSPHATE. Johnson \& Gwinn, Agents, Alderson, W. Va. Guarantee: (As above.) Found: Phosphoric acid, soluble, II.58; reverted, 3.95 ; insoluble, 0.38 ; total, I 5.8I ; available, I 5.43 .

5468. ZELL'S DISSOLVED S. C. PHOSPHATE. Benson Jacobs, Agent, Little Falls, W. Va. Guarantee: Phosphoric acid, soluble, Io; reverted, 2 ; insoluble, I ; total, I3; available, I2; phosphoric acid from dissolved phosphate rock. Found: Phosphoric acid, soluble, 7.25; reverted, 5.9I insoluble, 0.8I ; total, I4.97; available, I4. I6.

5634. ZELL'S DISSOLVED S. C. PHOSPHATE. Ben- 
son Jacobs, Agent, Little Falls, W. Va. Guarantee: (As above.) Found: Phosphoric acid, soluble, 6; reverted, 7.50; insoluble, I.07; total, I4.57; available, I3.50.

.5469. ZELL'S ELECTRIC PHOSPHATE. Benson Jacobs, Agent, Little Falls, W. Va. Guarantee: Phosphoric acid, soluble, 8 ; reverted, 2 ; insoluble, I ; total, I I; available, IO; potash, 2 ; phosphoric acid from dissolved phosphate rock; potash from kainit. Found: Phosphoric acid, soluble, 6.I9; reverted, 4.55 ; insoluble, o.67; total, II.4I ; available, Io.74; potash, $2 . \mathrm{I} 2$; chlorine, 2.9 .

5520. ZELL'S ELECTRIC PHOSPHATE. Philippi Mill Co., Agent, Philippi, W. Va. Guarantee: (As above.) Found: Phosphoric acid, soluble, 5.76; reverted, 3.I5; insoluble, I.64; total, ro.55; available, 8.91 ; potash, 2.16; chlorine, 5.5 .

Remark: Chlorine excessive.

5602. ZELL'S ELECTRIC PHOSPHATE. Exchange Mill Co., Agent, Grafton, W. Va. Guarantee: (As above.) Found: Phosphoric acid, soluble, 5.66; reverted, 4.72; insoluble, 0.36 ; total, I0.64; available, I0.28; potash, 2.30 ; chlorine, 2.9.

5625. ZELL'S ELECTRIC PHOSPHATE. Cicero Philipps, Agent, Belington, W. Va. Guarantee: (As above.) Found: Phosphoric acid, soluble, 3.10; reverted, 7.73; insoluble, 0.75 ; total, I I.68; available, Io.83 ; potash, 2.34 ; chlorine, 2.5 .

5642. ZELL'S ELECTRIC PHOSPHATE. Benson Jacobs, Agent, Little Falls, W. Va. Guarantee: (As above.) Found: Phosphoric acid, soluble, 7.63 ; reverted, 2.80 ; insoluble, 0.47 ; total, I0.90; available, I0.43; potash, 2.33 ; chlorine, 2.7 .

5737. ZELL'S ELECTRIC PHOSPHATE. Philippi Mill Co., Agent, Philippi, W. Va. Guarantee: (As above.) Found: Phosphoric acid, soluble, 7.68; reverted, 2.64; insoluble, 0.40 ; total, I0.72; available, I0.32; potash, 2.15 ; chlorine, 3.I. 
5606. ZELL'S SPECIAL COMPOUND FOR POTATOES AND VEGETABLES. Exchange Mill Co., Agent, Grafton, W. Va. Guarantee: Phosphoric acid, soluble, 6; reverted, 2; insoluble, I ; total, 9; available, 8 ; nitrogen, 2.65; potash, 4; phosphoric acid from dissolved animal bone and dissolved phosphate rock; nitrogen not less than one-tenth from bone tankage, not less than one-tenth from fish, the remainder from base goods, made from animal matter, treated by our own process, potash from muriate of potash. Found: Phosphoric acid, soluble, 4.26; reverted, 3.73; insoluble, I.50; total, 9.49; available, 7.99; nitrogen, 2.59; potash, 4.37 ; availability of nitrogen, 84 ; chlorine, r.7.

Remark: Chlorine excessive.

5645. ZELL'S AMMONIATED BONE SUPERPHOSPHATE. C. R. Carmen, Agent, Wellsburg, W. Va. Guarantee: Phosphoric acid, soluble, 6; reverted, 2; insoluble, I; total, 9; available, 8 ; nitrogen, 1.65 ; potash, 2 ; phosphoric acid from dissolved animal bone and dissolved phosphate rock; nitrogen not less than one-tenth from fish, not less than one-tenth from bone tankage, the remainder from base goods, made from animal matter, treated by our own process; potash from kainit. Found: Phosphoric acid, soluble, 5.18; reverted, 3.98; insoluble, 1.87; total, Ir.o3; available, 9.16; nitrogen, r.46; potash, 2.25 ; availability of nitrogen, 93 ; chlorine, I.5.

Remark: Nitrogen low.

5736. ZELL'S AMMONIATED BONE SUPERPHOSPHATE. Philippi Mill Co., Agent, Philippi, W. Va. Guarantee: (As above.) Found: Phosphoric acid, soluble, 4.22; reverted, $3.4 \mathrm{I}$; insoluble, I.62; total, 9.25; available, 7.63; nitrogen, I.73; potash, 2.39; availability of nitrogen, 86 ; chlorine, 3.5 .

Remark: Chlorine excessive; available phosphoric acid low.

5769. ZELL'S AMMONIATED BONE SUPERPHOSPHATE. R. Hunter, Agent, Berkeley Springs, W. Va. Guarantee: (As above.) Found: Phosphoric acid, soluble, 4.29; 
reverted, 3.38; insoluble, I.94; total, 9.61; available, 7.67; nitrogen, 1.78; potash, 2.27; availability of nitrogen, 89; chlorine, 3.4 .

Remark: Available phosphoric acid low. Chlorine excessive.

\section{ABBO'T'T \& MARTIN RENDERING CO., Columbus, Ohio.}

5498. STAR PHOSPHATE. Stortz, Blazer \& Co., Agent, Point Pleasant, W. Va. Found: Phosphoric acid, soluble, r.or ; reverted, 8.87; insoluble, 3.8I ; total, 13.69; available, 9.88; potash, 1.76; chlorine, r.2.

564I. PEERLESS BONE AND POTASH. W. P. Mason $\&$ Co., Agent, Mannington, W. Va. Found: Phosphoric acid, soluble, 0.77; reverted, 7.19; insoluble, 2.70; total, Io.66; available, 7.96 ; nitrogen, .56; potash, 3.09 ; availability of nitrogen, .68 ; chlorine, 1.3 .

ARMOUR FERTILIZER WORKS.

BALTIMORE, MD.

5522. BONE MEAL. A. G. Chrislip, Agent, Philippi, W. Va. Guarantee: Phosphoric acid, total, 24; nitrogen, 2.47; phosphoric acid from animal bone; nitrogen from bone. Found: Phosphoric acid, total, 24.78; nitrogen, 2.68 ; availability of nitrogen, 92.

5669. BONE MEAL. C. E. Baylor, Agent, Charles Town, W. Va. Guarantee: (As above.) Found: Phosphoric acid, total, 24.36; nitrogen, 2.55 ; availability of nitrogen, 93 . 
5667. GENUINE GERMAN KAINIT. C. E. Baylor, Charles Town, W. Va. Guarantee: Potash, 12; potash from kainit. Found: Potash, I2.83; chlorine, 2.9.

563r. GRAIN GROWER. Submitted for analysis by John Robinson, West Alexandria, Pa. Guarantee: Phosphoric acid, soluble, 6; reverted, 2 ; insoluble, 2 ; total, Io; available, 8; nitrogen, I.65; potash, 2 ; phosphoric acid from acid phosphate and bone; nitrogen, one-tenth to two-tenths bone, eight-tenths to nine-tenths bone tankage; potash, one-fifth to two-fifths from muriate, three-fifths to four-fifths from kainit. Found: Phosphoric acid, soluble, 5.09; reverted, 4.68; insoluble, 1.82 ; total II.58; available, 9.77 ; nitrogen, I.57 ; potash, 2.31 ; availability of nitrogen, 84 ; chlorine, 2.8 .

5671. RAW BONE MEAL. C. E. Baylor, Agent, Charles Town, W. Va. Guarantee: Phosphoric acid, total, 22 ; nitrogen, 3.70; phosphoric acid from bone; nitrogen from bone. Found: * Phosphoric acid, total, 22.03; nitrogen, 3.95; availability of nitrogen, 8r.

5707. RAW BONE MEAL. Parker, Smith \& Ours, Agent, Petersburg, W. Va. Guarantee: (As above.) Found: Phosphoric acid, total, 22.22 ; nitrogen, 3.48 ; availability of nitrogen, 70.

Remark: Nitrogen low.

5673. I6\% ACID PHOSPHATE. C. E. Baylor, Agent, Charles Town, W. Va. Found: Phosphoric acid, soluble, I4.or ; reverted, 2.65 ; insoluble, 0.43 ; total, I7.09; available, I6.66.

5679. ARMOUR'S TANKAGE. C. E. Baylor, Agent, Charles Town, W. Va. Found: Phosphoric acid, total, 6.I7; nitrogen, 5.65; availability of nitrogen, 97.

5709. STAR PHOSPHATE. Parker, Smith \& Ours, Agent, Petersburg, W. Va. Guarantee: Phosphoric acid, soluble, I2; reverted, 2 ; insoluble, 2 ; total, 16 ; available, I4; phosphoric acid 
from acid phosphate. Found: Phosphoric acid, soluble, I2.60; reverted, 2.89; insoluble, .78; total, 16.27; available, I 5.49.

5768. STAR PHOSPHATE. A. R. Unger, Agent, Berkeley Springs, W. Va. Guarantee: (As above.) Found: Phosphoric acid, soluble, I2.47; reverted, 4.34; insoluble, .63; total, 17.44 ; available, I6.81.

5785. STAR PHOSPHATE. Submitted for analysis by Josephus Jones, Morgantown, W. Va. Guarantee: (As above.) Found: Soluble, I2.03; reverted, 2.46; insoluble, .84; total, I 5.33 ; available, I4.47.

5708. PHOSPHATE AND POTASH NO. I. Parker, Smith \& Ours, Agent, Petersburg, W. Va. Guarantee: Phosphoric acid, soluble, 8 ; reverted, 2 ; insoluble, 2 ; total, 12 ; available, ro; potash, 2 ; phosphoric acid from acid phosphate; potash, one-fifth to two-fifths from muriate, three-fifths to four-fifths fron kainit. Found: Phosphoric acid, soluble, 6.32; reverted, 3.I I insoluble, I.or ; total, I0.44; available, 9.43; potash, 2.32; chlorine, 3.2.

Remark: Chlorine excessive.

5719. ROYAL AMMONIATED BONE. Parker, Smith \& Ours, Agent, Petersburg, W. Va. Guarantee: Phosphoric acid, soluble, 6 ; reverted, 2 ; insoluble, 2 ; total, Io; available, 8 ; nitrogen, 0.82 ; potash, 4; phosphoric acid from acid phosphate and animal bone; nitrogen, one-fifth to three-fifths from bone tankage, two-fifths to four-fifths from animal bone; potash, one-third to two-thirds from kainit; one-third to two-thirds from muriate. Found: Phosphoric acid, soluble, 5.21 ; reverted, 2.I4; insoluble, I.27; total, 9.62; available, 8.35; nitrogen, .95; potash, 4.60; availability of nitrogen, 82 ; chlorine, 2.7 . 


\section{BAUGH \& SONS CO.,}

BaLtTIMORE, MD.

5505. BAUGH'S EXCELSIOR CORN AND PEANUT GUANO. Conaway \& Clayton, Agent, Barracksville, W. Va. Guarantee: Phosphoric acid, soluble, 4; reverted, 4; insoluble, 2; total, Io; available, 8 ; nitrogen, 0.80 ; potash, 4; phosphoric acid from animal matter; nitrogen, from animal matter; potash from manure salts. Found: Phosphoric acid, soluble, 6.13; reverted, I.88; insoluble, 78 ; total, 8.79 ; available, 8.01 ; nitrogen, 0.97 ; potash, 4.94 ; availability of nitrogen, 64 ; chlorine, 1.7.

5558. BAUGH'S EXCELSIOR CORN AND PEANUT GUANO. Dean \& Reeves, Agent, Fairmont, W. Va. Guarantee: (As above.) Found: Phosphoric acid, soluble, 4.64; reverted, 3.88 ; insoluble, 0.75 ; total, 9.27 ; available, 8.52 ; nitrogen, I.03; potash, 3.88; availability of nitrogen, 64 ; chlorine, 2.3 .

5600. BAUGH'S EXCELSIOR CORN AND PEANUT GUANO. R. T. Lowndes, Agent, Clarksburg, W. Va. Guarantee: (As above.) Found: Phosphoric acid, soluble, 6.09; reverted, $3.3 \mathrm{I}$; insoluble, I.45; total, I0.85; available, 9.40 ; nitrogen, I.3I ; potash, 4.22; availability of nitrogen, 77 ; chlorine, I.5.

5777. BAUGH'S EXCELSIOR. CORN AND PEANUT GUANO. A. P. Russell \& Co., Agent, Buckhannon, W. Va. Guarantee: (As above.) Found: Phosphoric acid, soluble, 4.99; reverted, 2.33; insoluble, 2.1o; total, 9.42; available, 7.32; nitrogen, I.25; potash, 4.77 ; availability of nitrogen, 86 ; chlorine, I.7.

Remark: Phosphoric acid low.

5506. BAUGH'S GENERAL CROP GROWER. Conaway \& Clayton, Agent, Barracksville, W. Va. Guarantee: Phosphoric acid, insoluble, 2.5 ; total, 10.5; available, 8 ; nitrogen, .80; potash, I; phosphoric acid from animal matter; nitrogen, from 
animal matter; potash from manure salt. Found: Phosphoric acid, soluble, 4.9I ; reverted, 3.45; insoluble, 0.99; total, 9.35; available, 8.36 ; nitrogen, I.20; potash, I.60; availability of nitrogen, 60 ; chlorine, 2.9 .

Remark: Chlorine excessive.

5549. BAUGH'S GENERAL CROP GROWER. T. P. Licklider, Agent, Martinsburg, W. Va. Guarantee: (As above.) Found: Phosphoric acid, soluble, 5.77 ; reverted, 2.38; insoluble, 0.50; total, 8.65 ; available, 8.I5; nitrogen, I.70; potash, I.83; availability of nitrogen, 76 ; chlorine, 2.2 .

5595. BAUGH'S GENERAL CROP GROWER. R. T. Lowndes, Agent, Clarksburg, W. Va. Guarantee: (As above.) Found: Phosphoric acid, soluble, 6.08; reverted, 3.35 ; insoluble, I.46; total, Io.89; available, 9.43 ; nitrogen, I.IO; potash, I.99; availability of nitrogen, 75 ; chlorine, 2.3 .

5653. BAUGH'S GENERAL CROP GROWER. T. P. Licklider, Agent, Martinsburg, W. Va. Guarantee: (As above.) Found: Phosphoric acid, soluble, 5.37; reverted, 3.88; insoluble, I.02 ; total, I0.27 ; available, 9.25 ; nitrogen, o.80; potash, I.84; chlorine, 2.8 .

5775. BAUGH'S GENERAL CROP GROWER. J. J. Lyne, Shenandoah Junction, W. Va. Guarantee: (As above.) Found: Phosphoric acid, soluble, 2.49; reverted, 5.26; insoluble, 1.93; total, 9.69; available, 7.75 ; nitrogen, 0.92 ; potash, I.44; availability of nitrogen, 70 ; chlorine, 3.2.

Remark: Chlorine excessive. Source of potash equivalent to kainit.

5507. BAUGH'S SPECIAL POTATO MANURE (IO\%) $P O T A S H$. Conaway \& Clayton, Agent, Barracksville, W. Va. Guarantee: Phosphoric acid, insoluble, 2.50; total, 7.50; available, 5 ; nitrogen, I.60; potash, Io; phosphoric acid from animal matter; nitrogen, from animal matter; potash, $1 / 4$ from muriate of potash, $1 / 4$ from sulphate of potash. Found: Phosphoric acid, 
soluble, I.5I ; reverted, 3.38 ; insoluble, 2.80 ; total, 7.69; available, 4.89; nitrogen, I.39; potash, I0.38; availability of nitrogen, 77 ; chlorine, I.2.

Remark: Nitrogen low.

5556. BAUGH'S SPECIAL POTATO MANURE (IO\%) POTASH. Dean \& Reeves, Agent, Fairmont, W. Va. Guarantee: (As above.) Found: Phosphoric acid, soluble, 2.II; reverted, 4.01 ; insoluble, I.Io; total, 7.22 ; available, 6.I2 ; nitrogen, r.69; potash, 8.57 ; availability of nitrogen, 82 ; chlorine, 1.2.

Remark: Potash low.

5578. BAUGH'S SPECIAL POTATO MANURE (IO\%) POTASH. West Virginia Implement Co., Agent, Elkins, W. Va. Guarantee: (As above.) Found: Phosphoric acid, soluble, I.56; reverted, 3.75; insoluble, I.09; total, 6.40; available, 5.3I ; nitrogen, I.74; potash, 9.79; availability of nitrogen, 86; chlorine, I.2.

5508. BAUGH'S HIGH GRADE ACID PHOSPHATE ROCK. Conaway \& Clayton, Agent, Barracksville, W. Va. Guarantee: Phosphoric acid, available, I4; phosphoric acid from dissolved Florida rock. Found: Phosphoric acid, soluble, 12.I9; reverted, 3.54 ; insoluble, 0.45 ; total, I6.I8; avaiłable, I 5.73.

5546. BAUGH'S HIGH GRADE ACID PHOSPHATE ROCK. T. P. Licklider, Agent, Martinsburg, W. Va. Guarantee: (As above.) Found: Phosphoric acid, soluble, 9.70; reverted, 4.99; insoluble, r.33; total, I6.02 ; available, I4.69.

5609. BAUGH'S HIGH GRADE ACID PHOSPHATE ROCK. West Virginia Implement Co., Agent, Elkins, W. Va. Guarantec: (As above.) Found: Phosphoric acid, soluble, I 2.42 ; reverted, 4.28 ; insoluble, 0.75 ; total, I7.45; available, I6.70.

5774. BAUGH'S HIGH GRADE ACID PHOSPHATE. J. H. Phillips \& Sons, Berkeley Springs, W. Va. Guarantee: 
(As above.) Found: Phosphoric acid, soluble, II.26; reverted, 5.22 ; insoluble, I.92; total, I8.40; available, I6.48.

5509. BAUGH'S ANIMAL BONE AND POTASH COMPOUND. Conaway \& Clayton, Agent, Barracksville, W. Va. Guarantee: 'Phosphoric acid, insoluble, 2.50; total, IO.50; available, 8; nitrogen, I.6o; potash, 2 ; phosphoric acid from animal matter; nitrogen from animal matter; potash from manure salt. Found: Phosphoric acid, soluble, 4.70; reverted, 3.4I ; insoluble, I.68; total, 9.79; available, 8.II; nitrogen, I.49; potash, 2.50; availability of nitrogen, 62 ; chlorine, 2.

Remark: Nitrogen low.

559I. BAUGH'S ANIMAL BONE AND POTASH COMPOUND. R. T. Lowndes, Agent, Clarksburg, W. Va. Guarantee: (As above.) Found: Phosphoric acid, soluble, 5.59; reverted, 2.49; insoluble, 2.19; total, I0.27; available, 8.08 ; nitrogen, 2. I I potash, 2.97 ; availability of nitrogen, 84 ; chlorine, I.4.

5544. BAUGH'S POTATO FERTILIZER. T. P. Licklider, Agent, Martinsburg, W. Va. Found: Phosphoric acid, soluble, 1.69; reverted, 3.I3; insoluble, 2.56; total, 7.38; availabie, 4.82 ; nitrogen, I.70; potash, 6.I4; availability of nitrogen, $8 \mathrm{I}$; chlorine, 2.2.

5545. BAUGH'S RAW BONE SUPERPHOSPHATE. T. P. Licklider, Agent, Martinsburg, W. Va. Found: Phosphoric acid, soluble, 4.77 ; reverted, $3.5^{\mathrm{I}}$; insoluble, 0.99; total, 9.27 ; available, 8.28 ; nitrogen, 1.60; potash, 1.80; availability of nitrogen, 84 ; chlorine, 3.x.

5555. BAUGH'S DOUBLE EAGLE \$25 PHOSPHATE AND A RAW BONE SUPERPHOSPHATE. Dean \& Reeves, Agent, Fairmont, W. Va. Guarantee: Phosphoric acid, soluble, 2.50 ; total, I0.50; available, 8 ; nitrogen, I.60; potash, I ; phosphoric acid from animal matter; nitrogen from anımal matter; potash from manure salt. Found: Phosphoric acid, soluble, 4.32 ; reverted, 3.78; insoluble, o.87; total, 8.97; available, 8.Io; 
nitrogen, r.6I ; potash, r.49; availability of nitrogen, 85 ; chlorine, 2.8 .

Remark: Chlorine high.

5592. BAUGH'S DOUBLE EAGLE \$25 PHOSPHATE AND $A$ RAW BONE SUPERPHOSPHATE. R. T. Lowndes, Agent, Clarksburg, W. Va. Guarantee: (As above.) Found: Phosphoric acid, soluble, 6.32; reverted, 2.36; insoluble, r.89; total, I0.57; available, 8.68 ; nitrogen, I.98; potash, 1.96; availability of nitrogen, 70 ; chlorine, 2.2 .

5663. BAUGH'S DOUBLE EAGLE \$25 PHOSPHATE $A N D$ A RAW BONE SUPERPHOSPHATE. N. C. Musgrave, Agent, Grafton, W. Va. Guarantee: (As above.) Found: Phosphoric acid, soluble, 2.05; reverted, 6.57; insoluble, I.23; total, 9.85 ; available, 8.62 ; nitrogen, I.72 ; potash, I.42 ; availability of nitrogen, 89 ; chlorine, 2.3 .

5758. BAUGH'S DOUBLE EAGLE \$25 PHOSPHATE AND A RAW BONE SUPERPHOSPHATE. G. T. Hodges, Agent, Shepherdstown, W. Va. Guarantee: (As above.) Found: Phosphoric acid, soluble, 6.09; reverted, 2.65; insoluble, r.39; total, I0.03; available, 8.64 ; nitrogen, r.84; potash, 1.44; availability of nitrogen, 75 ; chlorine, 2.9 .

5557. BAUGH'S POTATO AND TRUCK SPECIAL. Dean \& Reeves, Agent, Fairmont, W. Va. Guarantee: Phosphoric acid, insoluble, 2.50; total, 9.50; available, 7 ; nitrogen, 2.80; potash, 7 ; phosphoric acid from animal matter; nitrogen from animal matter; potash from muriate of potash. Found: Phosphoric acid, soluble, 4.98; reverted, 2.03; insoluble, .83; total, 7.84; available, 7.or ; nitrogen, 2.49 ; potash, 6.94; availability of nitrogen, $8 \mathrm{I}$; chlorine, .7 .

Remark: Nitrogen low.

5222. (G. R. B.). BAUGH'S POTATO AND TRUCK $S P E C I A L$. Submitted for analysis by J. D. Bowman, Hoult, W. Va. Guarantee: (As above.) Found: Phosphoric acid, 
soluble, 5.12 ; reverted, 2.16 ; insoluble, I.04; total, 8.32 ; available, 7.29; nitrogen, 2.79; potash, 7.29.

5571. BAUGH'S PURE BONE MEAL. West Virginia, Implement Co., Agent, Elkins, W. Va. Guarantee: Phosphoric acid, total, $2 \mathrm{I}$; nitrogen, 3.60 ; phosphoric acid from pure bone; nitrogen from pure bone. Found: Phosphoric acid, total, 22.49; nitrogen, 3.62 ; availability of nitrogen, 91 .

5604. BAUGH'S PURE BONE MEAL. R. T. Lowndes, Agent, Clarksburg, W. Va. Guarantee: (As above.) Found: Phosphoric acid, total, 20.47 ; nitrogen, 3.97 ; availability of nitrogen, 78 .

Remark: Phosphoric acid low.

5652. BAUGH'S PURE DISSOLVED ANIMAL BONE. T. P. Licklider, Agent, Martinsburg, W. Va. Guarantee: Phosphoric acid, available, I3; nitrogn, 2 ; phosphoric acid from sulphuric acid and animal bone; nitrogen from pure bone. Found: Phosphoric acid, total, 20.12 ; nitrogen, 2.86 ; availability of nitrogen, 9I.

5757. BAUGH'S PURE DISSOLVED ANIMAL BONE: G. T. Hodges, Agent, Shepherdstown, W. Va. Guarantee: (As above.) Found: Phosphoric acid, total, I8.74; nitrogen, 2.39; availability of nitrogen, 92 .

5657. BAUGH'S COMPLETE ANIMAL, BONE FERTILIZER. T. P. Licklider, Agent, Martinsburg, W. Va. Guarantee: Phosphoric acid, insoluble, 2; total, Io; available, 8; nitrogen, I.6o; potash, 5; phosphoric acid from animal matter; nitrogen from animal matter; potash from manure salt. Found: Phosphoric acid, soluble, 4.48; reverted, 3.72; insoluble, I.54; total, 9.74; available, 8.20 ; nitrogen, 1.66; potash, 4.93; availability of nitrogen, 83 ; chlorine, I.

5658. BAUGH'S SOLUBLE ALKALINE. T. P. Licklider, Agent, Martinsburg, W. Va. Guarantee: Phosphoric 
acid, available, Io; potash, 2; phosphoric acid from dissolved Florida rock; potash from manure salt. Found: Phosphoric acid, soluble, 2.30; reverted, 8.03; insoluble, I.80; total, I2.I3; available, I0.33; potash, 2.35 ; chlorine, 5.9.

Remark: Source of potash equivalent to kainit; chlorine excessive.

5756. BAUGH'S ONE-QUARTER MIXTURE. G. T. Hodges, Agent, Shepherdstown, W. Va. Found: Phosphoric acid, soluble, 2.92 ; reverted, 7.82 ; insoluble, 2.I4; total, I2.88; available, I0.74; nitrogen, I.52 ; potash, 3.OI ; availability of nitrogen, 89; chlorine, 2.7 .

\section{BAUGH'S EXPORT BONE WITH POTASH. G.} T. Hodges, Agent, Shepherdstown, W. Va. Guarantee: Phosphoric acid, total, II ; nitrogen, I.6o; potash, 2.; phosphoric acid from pure bones; nitrogen from pure bones; potash from manure salt. Found: Phosphoric acid, soluble, trace; reverted, 6.96; insoluble, 4.45 ; total, II.4I ; available, 6.96 ; nitrogen, I.85; potash, 2.66; availability of nitrogen, 74 ; chlorine, 2.3 .

5776. BAUGH'S AMMONIATED SOLUBLE ALKALI. A. P. Russell \& Co., Agent, Buckhannon, W. Va. Guarantee: Phosphoric acid, available, 8; nitrogen, .40; potash, 2; phosphoric acid from dissolved Florida rock; nitrogen from animal matter; potash from manure salt. Found: Phosphoric acid, soluble, 2.69 ; reverted, 7.I4 ; insoluble, 0.46; total, I0.29; available, 9.83 ; nitrogen, 0.43 ; potash, 2.59 ; availability of nitrogen, 78 ; chlorine, I.9.

5778. BAUGH'S PENINSULA GRAIN PRODUCER. A. P. Russell \& Co., Agent, Buckhannon, W. Va. Guarantee: Phosphoric acid, insoluble, 2; total, Io; available, 8; nitrogen, o.80; potash, 2; phosphoric acid from animal matter; nitrogen from animal matter; potash from manure salt. Found: Phosphoric acid, soluble, I.92; reverted, 6.92; insoluble, I.69; total, I0.53; available, 8.84 ; nitrogen, 0.93 ; potash, 2.63 ; availability of nitrogen, 67 ; chlorine, 2.5 . 
5627. NITRATE OF SODA...Dean \& Reeves, Agent, Fairmont, W. Va. Found: Nitrogen, 15.73.

5628. MURIATE OF POTASH. Dean \& Reeves, Agent, Fairmont, W. Va. Found: Potash, 48.90; chlorine, .9.

BALTIMORE PULVERIZING CO.,

BALTTMORE, MD.

5668. SPECIAL FALL MIXTURE. Washington \& Alexander, Agent, Charles Town, W. Va. Guarantee: Phosphoric acid, insoluble, I.50; total, 9.50 ; available, 8.00 ; potash, I ; phosphoric acid from Florida phosphate; potash from sulphate of potash. Found: Phosphoric acid, soluble, 0.64; reverted, 7.22; insoluble, I.80; total, 9.66; available, 7.86; potash, I.20; chlorine, 3 .

Remark: Source of potash equivalent to kainit.

5686. SPECIAL FALL MIXTURE. R. N. Stewart \& Son, Agent, Martinsburg, W. Va. Guarantee: (As above.) Found: Phosphoric acid, soluble, 0.77; reverted, 9.06; insoluble, 2.26; total, I2.09; available, 9.83 ; potash, I.56; chlorine, 3.6.

Remark; Source of potash equivalent to kainit. Chlorine excessive.

5569. BOWKER'S HARVEST BONE. P. H. Wees \& Son, Agent, Elkins, W. Va. Guarantee: Phosphoric acid, insoluble, 2 ; total, Io ; available, 8 ; nitrogen, 0.82 ; potash, I ; phosphoric acid from phosphate rock and animal tankage; nitrogen from animal tankage; potash from muriate. Found: Phosphoric acid, soluble, 4.07 ; reverted, 4.59 ; insoluble, 2.85 ; total, II.5I ; available, 8.66; nitrogen, I.I2; potash, I.42; availability of nitrogen, 82 ; chlorine, $\mathrm{I}$. 


\section{BOWKER FERTILIZER CO.,}

Cincinnati, Ohio.

5570. BOWKER'S HARVEST BONE. West Virginia Implement Co., Agent, Elkins, W. Va. Guarantee: (As above.) Found: Phosphoric acid, soluble, I.I5; reverted, 7.14; insoluble, 2.74 ; total, II.03; available, 8.29; nitrogen, 0.9I ; potash, I.08; availability of nitrogen, 67 ; chlorine, I.9.

Remark: Chlorine high.

5594. BOWKER'S HARVEST BONE. Orie Myers, Agent, Clarksburg, W. Va. Guarantee: (As above.) Found: Phosphoric acid, soluble, 3.22; reverted, 6.38; insoluble, 2.46; total, I2.06; available, 9.60 ; nitrogen, I.I2; potash, I.40; availability of nitrogen, 76 ; chlorine, ז.2.

56I I. BOWKER'S HARVEST BONE. W. F. Sill, Agent, Pennsboro, W. Va. Guarantee: (As above.) Found: Phosphoric acid, soluble, 3.84 ; reverted, 5.46; insoluble, 2.37 ; total, II.67; available, 8.30 ; nitrogen, 0.97 ; potash, I.30; availability of nitrogen, 77 ; chlorine, .8 .

56I5. BOWKER'S HARVEST BONE. T. B. Drummond \& Co., Agent, Buckhannon, W. Va. Guarantee: (As above.) Found: Phosphoric acid, soluble, 2.84; reverted, 5.88; insoluble, 2.32 ; total, II.04; available, 8.72 ; nitrogen, I.04; potash, I.25; availability of nitrogen, 70 ; chlorine, I.I.

5574. BOWKER'S SOLUBLE BONE. P. H. Wees \& Son, Agent, Elkins, W. Va. Guarantee: Phosphoric acid, insoluble, 2 ; total, I6; available, I4; phosphoric acid from phosphate rock.' Found: Phosphoric acid, soluble, 9.80; reverted, 5.93; insoluble, 2.44 ; total, I8.17; available, I5.73.

560I. BOWKER'S SOLUBLE BONE. W. F. Sill, Agent, Pennsboro, W. Va. Guarantee: (As above.) Found: Phos-- 
phoric acid, soluble, 8.83 ; reverted, 8.69 ; insoluble, I.22; total, I8.74; available, $\mathbf{I 7 . 5 2}$.

5740. BOWKER'S SOLUBLE BONE. A. G. Chrislip, Agent, Philippi, W. Va. Guarantee: (As above.) Found: Phosphoric acid, soluble, 9.27; reverted, 5.99; insoluble, 0.4I; total, I 5.67 ; available, I 5.26 .

5575. BOWKER'S DISSOLVED BONE WITH POT$A S H . \quad$ P. H. Wees \& Son, Agent, Elkins, W. Va. Guarantee: Phosphoric acid, insoluble, 2 ; total, I2 ; available, IO; potash, 2; phosphoric acid from phosphate rock; potash from muriate. Found: Phosphoric acid, soluble, 4.82 ; reverted, 6.4I ; insoluble, 2.75 ; total I3.98; available, II.23; potash, I.90; chlorine, I.2.

5608. BOWKER'S DISSOLVED BONE WITH POT$A S H$. Orie Myers, Agent, Clarksburg, W. Va. Guarantee: (As above.) Found: Phosphoric acid, soluble, 4.02 ; reverted, 7.29; insoluble, 2.76; total, I4.07; available, II.3I ; potash, 2; chlorine, I.I.

56I3. BOWKER'S DISSOLVED BONE WITH POT$A S H$. T. B. Drummond \& Co., Agent, Buckhannon, W. Va. Guarantee: (As above.) Found: Phosphoric acid, soluble, 3.83 ; reverted, 6.64 ; insoluble, 3.08 ; total, I3.55; available, 9.47 ; potash, 2.16 ; chlorine, 1.

5783. BOWKER'S DISSOLVED BONE WITH POTASH. T. B. Drummond \& Co., Agent, Buckhannon, W. Va. Guarantee: (As above.) Found: Phosphoric acid, soluble, 2.62 ; reverted, 7.71 ; insoluble, 3.6I ; total, I3.94; available, I0.33; potash, 2.01 ; chlorine, I.I.

5580. BOWKER'S POTASH FERTILIZER. P. H. Wees \& Son, Agent, Elkins, W. Va. Guarantee: Phosphoric acid, insoluble, 2; total, I2; available, IO; potash, 5; phosphoric acid from phosphate rock; potash from muriate. Found: Phos- 
phoric acid, soluble, 3.62 ; reverted, 6.35 ; insoluble, 2.26 ; total, I2.23; available, 9.97 ; potash, 4.82 ; chlorine, I.I.

5599. BOWKER'S POTASH FERTILIZER. T. B. Drummond \& Co., Agent, Buckhannon, W. Va. Guarantee: (As above.) Found: Phosphoric acid, soluble, 6.03; reverted, 5.02; insoluble, 2.I4; total, I3.I9; available, II.05; potash, 4.74; chlorine, I.

5607. BOWKER'S POTASH FERTILIZER. Orie Myers, Agent, Clarksburg, W. Va. Guarantee: (As above.) Found: Phosphoric acid, soluble, 3.38; reverted, 6.75; insoluble, 2.09; total, I2.22; available, IO.I3; potash, 5.27; chlorine, I.I.

5620. BOWKER'S POTASH FERTILIZER. J. M. Miller \& Bro., Agent, Ronceverte, W. Va. Guarantee: (As above.) Found: Phosphoric acid, soluble, 3.74; reverted, 6.46; insoluble, 2.53; total, I2.73; available, ro.20 ; potash, 5.57 ; chlorine, .9.

574I. BOWKER'S POTASH FERTILIZER. A. G. Chrislip, Agent, Philippi, W. Va. Guarantee: (As above.) Found: Phosphoric acid, soluble, 5.56; reverted, 4.34 ; insoluble, I.05; total, I0.95; available, 9.90 ; potash, 4.74 ; chlorine, 2.2.

Remark: Source of potash equivalent to manure salt.

57I5. BOWKER'S POTASH FERTILIZER. J. M. Mi1ler \& Bro., Agent, Ronceverte, W. Va. Guarantee: (As above.) Found: Phosphoric acid, soluble, 5.82 ; reverted, 4.60; insoluble, 2.56 ; total, I2.98; available, I0.42; potash, 4.74 ; chlorine, I.3.

5727. BOWKER'S POTASH FERTILIZER. John Gay, Agent, Marlinton, W. Va. Guarantee: (As above.) Found: Phosphoric acid, soluble, 5.37; reverted, 4.59; insoluble, $2.7 \mathrm{I}$; total, I2.67; available, 9.96 ; potash, 3.82 ; chlorine, I.I.

5782. BOWKER'S POTASH FERTILIZER. 'T. B. Drummond \& Co., Agent, Buckhannon, W. Va. Guarantee: (As above.) Found. Phosphoric acid, soluble, 3.84; reverted, 6.2I ; 
insoluble, 3.40 ; total, I3.45; available, I0.05 ; potash, 4.58 ; chlorine, r.r.

5590. BOWKER'S IO\% MANURE. Orie Myers, Clarksburg, W. Va. Guarantee: Phosphoric acid, insoluble, 2; total, 7 ; available, 5 ; nitrogen, .82; potash, Io; phosphoric acid from phosphate rock and animal tankage; nitrogen from animal tankage; potash from muriate. Found: Phosphoric acid, soluble, I.85; reverted, 4.27 ; insoluble, I.94; total, 8.06; available, 6.I2; nitrogen, 0.90; potash, I2.I4; availability of nitrogen, 77 ; chlorine, I.8.

Remark: Chlorine high.

5605. BOWKER'S BONE MEAL. Orie Myers, Agent, Clarksburg, W. Va. Guarantee: Phosphoric acid, total, 25; nitrogen, 1.23; phosphoric acid from bone; nitrogen from bone. Found: Phosphoric acid, total, 25.74; nitrogen, r.83; availability of nitrogen, 89 .

56I2. BOWKER'S BONE MEAL. T. B. Drummond \& Co., Agent, Buckhannon, W. Va. Guarantee: (As above.) Found: Phosphoric acid, total, 26.6r ; nitrogen, I.36; availability of nitrogen, 92 .

57I3. BOWKER'S BONE MEAL. J. M. Miller \& Bro., Agent, Ronceverte, W. Va. Guarantee: (As above.) Found: Phosphoric acid, total, 27.65 ; nitrogen, I.24; availability of nitrogen, 93 .

5629. MURIATE OF POTASH. T. B. Drummond \& Co., Agent, Buckhannon, W. Va. Found: Potash, 49.10; chlorine, .8.

5630. NITRATE OF SODA. T. B. Drummond \& Co., Agent, Buckhannon, W. Va. Found: Nitrogen, I5.83. 


\section{THE BRYANT FERTILIZER CO.,}

AleXANDRIa, Va.

5760. BRYANT'S S. C. DISSOLVED BONE. W. N. Lemen \& Son, Agent, Shepherdstown, W. Va. Guarantee: Phosphoric acid, total, I5.20; available, I4; phosphoric acid from Tennessee rock. Found: Phosphoric acid, soluble, II.55; reverted, 2.92 ; insoluble, 0.23 ; total, 15.70 ; available, 15.47 .

5761. BRYANT"S "QUEEN" AMMONIATED DISSOLVED BONE. W. N. Lemen \& Son, Agent, Shepherdstown, W. Va. Guarantee: Phosphoric acid, available, 8 ; nitrogen, .82 ; potash, $\mathrm{r}$; phosphoric acid from Tennessee rock; nitrogen from meat tankage, potash from kainit. Found: Phosphoric acid, soluble, 5.12; reverted, 3.72; insoluble, I.I9; total, 10.03; available, 8.84 ; nitrogen, I.08; potash, I ; availability of nitrogen, 60 ; chlorine, 2.

\section{CINCINNATI PHOSPHATE CO.,}

Cincinnati, Ohic.

5473. CAPITAL GRAIN AND GRASS GROWER. J. M. Rogers, Agent, Ronceverte, W. Va. Found: Phosphoric acid, soluble, 7.I8; reverted, 3.21 ; insoluble, 1.88 ; total, 12.27 ; available, 10.39; nitrogen, 0.87 ; potash, I.05; availability of nitrogen, 83 ; chlorine, .07.

5495. CAPITAL GRAIN AND GRASS GROWER. Damron Seed \& Feed Co., Agent, Huntington, W. Va. Found: Phosphoric acid, soluble, 7.25; reverted, 3.42; insoluble, 2.03; total, I2.70; available, I0.67; nitrogen, 0.89 ; potash, I.02; availability of nitrogen, 85 ; chlorine, .09. 
5726. CAPITAL WHEAT GROWER. Marlinton Supply Co., Agent, Marlinton, W. Va. Found: Phosphoric acid, soluble, 9.98; reverted, 3.78; insoluble, 2.90; total, 16.66; available, 13.76 .

\section{CHICAGO FERTILIZER CO.,}

Chicago, Ill.

5638. BONE, BLOOD AND POTASH. W. P. Mason \& Co., Agent, Mannington, W. Va. Guarantee: Phosphoric acid, soluble, 5 ; reverted, 2 ; total, Io; available, 8; nitrogen, I.23; potash, 2 ; phosphoric acid from dissolved bone phosphate; nitrogen, $1 / 4$ to $I / 2$ from bone tankage, $I / 4$ to $1 / 2$ from blood, $1 / 4$ to $I / 2$ from tobacco stems, $1 / 4$ to $T / 2$ from fish scrap; potash, $I / 2$ to $3 / 4$ from muriat of potash, $1 / 2$ to $3 / 4$ from tobacco stems. Found: Phosphoric acid, soluble, I.23; reverted, 7.06; insoluble, 3.40; total, Ir.69; available, 8.29; nitrogen, 0.92 ; potash, I.88; availability of nitrogen, $7 \mathrm{I}$; chlorine, I.

Remark: Nitrogen low.

5499. WHEAT AND CORN SPECIAL. Stortz, Blazer Co., Pt. Pleasant, W. Va. Guarantee: Phosphoric acid, soluble, 4; reverted, 3 ; total, 9; available, 7 ; nitrogen, 82 ; potash, I; phosphoric acid from dissolved bone phosphate; nitrogen, onethird to two-thirds bone tankage, one-third to two-thirds blood, one-third to two-thirds tobacco stems; potash, $1 / 2$ to $3 / 4$ from muriate of potash, $1 / 2$ to $3 / 4$ from tobacco stems. Found: Phosphoric acid, soluble, 2.88; reverted, 5.04; insoluble, 3.93; total, I1.85; available, 7.92 ; nitrogen, 0.80 ; potash, I.26; availability of nitrogen, 67 ; chlorine, .3 .

Remark: Nitrogen low.

5644. CHICAGO BONE MEAL. W. P. Mason \& Co., Agent, Mannington, W. Va. Guarantee: Phosphoric acid, total, 
20 ; nitrogen, I.23; phosphoric acid from bone; nitrogen from bone. Found: Phosphoric acid, total, I9.18; nitrogen, I.52; availability of nitrogen, 86 .

Remark: Phosphoric acid low.

\title{
S. M. HESS \& BRO.,
}

\section{S. E. Corner Fourth and Chestnut Sts.,}

\author{
Philadeliphia, Pa.
}

5526. KEYSTONE BONE PHOSPHATE. F. A. Simpson, Agent, Philippi, W. Va. Guarantee: Phosphoric acid, soluble, 6 ; reverted, 2 ; insoluble, I ; total, 9 ; available, 8 ; nitrogen, .82 ; potash, I; phosphoric acid from acid phospate; nitrogen from slaughter house tankage; potash from muriate; Found: Phosphoric acid, soluble, 4.36; reverted, 3.60 ; insoluble, 1.28 ; total, 9.24 ; available, 7.96 ; nitrogen, 0.84 ; potash, I.27; availability of nitrogen, 75 ; chlorine, 2.5 .

Remark: Source of potash equivalent to kainit.

5527. SOLUBLE BONE AND POTASH. F. A. Simpson, Agent, Philippi, W. Va. Guarantee: Phosphoric acid, soluble, 8; reverted, 2 ; insoluble, I ; total, II ; available, Io; potash, 2; phosphoric acid from acid phosphate; potash from muriate. Found: Phosphoric acid, soluble, 5.90; reverted, 3.89; insoluble, 0.5I ; total, I0.30; available, 9.89 ; potash, 2.13 ; chlorine, $3 \cdot 5$.

Remark: Source of potash equivalent to kainit. Chlorine excessive.

5528. SOLUBLE BONE. F. A. Simpson, Agent, Philippi, W. Va. Guarantee: Phosphoric acid, soluble, 12; reverted, 2 ; insoluble, I ; total, I 5 ; available, I4; phosphoric acid from acid phosphate. Found: Phosphoric acid, soluble, II.27; reverted, 3.85 ; insoluble, I.09; total, I6.21 ; available, I5.I2. 
569I. SOLUBLE BONE. Poling Bros., Agent, Belington, W. Va. Guarantee: (As above.) Found: Phosphoric acid, soluble, 13.75 ; reverted, 4.07 ; insoluble, $0.4 \mathrm{I}$; total, 18.23 ; available, r7.82.

\section{JARECKI CHEMICAL, CO., \\ Sandusky and Cincinnati, Ohio.}

5572. FISH AND POTASH, TOBACCO AND POTATO FOOD. W. S. Stout, Agent, Harrisville, W. Va. Guarantee: Phosphoric acid, soluble, 6; reverted, 2; insoluble, 1 ; total, 9; available, 8 ; nitrogen, .82 ; potash, 4 ; phosphoric acid from mineral phosphate, bone and animal tankage; nitrogen from animal tankage; potash from muriate. Found: Phosphoric acid, soluble, 6.45 ; reverted, 3.25 ; insoluble, I.39; total, Ir.09; available, 9.70; nitrogen, 0.78 ; potash, 3.14; availability of nitrogen, 72 ; chlorine, 0.9 .

Remark: Potash low.

55I3. FISH AND POTASH, TOBACCO AND POTATO FOOD. Myne Brothers, Hurricane, W. Va. Guarantee: (As above.) Found: Phosphoric acid, soluble, 7.09; reverted, 2.36; insoluble, 1.91 ; total, Ir.36; available, 9.45 ; nitrogen, 0.74; potash, 2.82 ; availability of nitrogen, 80 ; chlorine, 0.7 .

Remark: Potash low.

5563. GROUND BONE. W. S. Stout, Agent, Harrisville, W. Va. Guarantee: Phosphoric acid, total, 20; nitrogen, 2.50; phosphoric acid, from bone; nitrogen, from bone. Found: Phosphoric acid, total, 22.77; nitrogen, 2.73; availability of nitrogen, 92 .

5725. NO. ONE FISH GUANO. Marlinton Supply Co., Agent, Marlinton, W. Va. Guarantee: Phosphoric acid, soluble, 
8 ; reverted, 2 ; insoluble, I ; total, I I ; available, Io; nitrogen, .82 ; potash, I; phosphoric acid from mineral phosphate, bone and animal tankage; nitrogen from animal matter; potash from muriate. Found: Phosphoric acid, soluble, 6.9I ; reverted, 3.51 ; insoluble, 2.23 ; total, I2.65 ; available, I0.42 ; nitrogen, .80 ; potash, I.48; availability of nitrogen, 86 ; chlorine, 0.2 .

\title{
MARIETTA BONE AND PHOSPHATE CO.,
}

\author{
MARIETTTA, OHIO.
}

5500. PURE RAW BONE. Walter Marshall, Agent, Wheeling, W. Va. Guarantee: Phosphoric acid, total, 20; nitrogen, 4; phosphoric acid from bone; nitrogen from bone. Found: Phosphoric acid, soluble, I.48; reverted, 4.47 ; insoluble, 2.98; total, 9.93; available, 6.95; nitrogen, 0.96; potash, 2.34; availability of nitrogen, 72 ; chlorine, 2.6 .

Remark: This sample is not as guaranteed.

566r. PURE RAW BONE. Walter Marshall, Agent, Wheeling, W. Va. Guarantee: (As above.) Found: Phosphoric acid, total, 18.54; nitrogen, 3.43 ; availability of nitrogen, 78 .

Remark: Phosphoric acid low; nitrogen low.

acid, total, Io; available, 8 ; nitrogen, I ; potash, I.50; phosphoric acid, $3 / 4$ acid, $1 / 4$ bone tankage; nitrogen, bone $1 / 4$ and tankage, $3 / 4,1 / 2$ garbage and $1 / 2$ bone tankage; potash from kainit. Found: Phosphoric acid, soluble, I.46; reverted, 4.83; insoluble, 3.24; total, 9.53; available, 6.29; nitrogen, I.09; potash, 2.57; availability of nitrogen, 80 ; chlorine, 2.6 .

Remark: Available phosphoric acid low

5664. CORN, OATS AND WHEAT GROWER. Walter Marshall, Agent, Wheeling, W. Va. Guarantee: (As above.) 
Found: Phosphoric acid, soluble, 2.17 ; reverted, 4.18; insoluble, 2.25; total, 8.60 ; available, 6.35 ; nitrogen, .9I ; potash, I.90; availability of nitrogen, 87 ; chlorine, 2.5 .

5504. ACID PHOSPHATE. C. F. Braumlich \& Co., Agent, Wheeling, W. Va. Guarantee: Phosphoric acid, available, I6; phosphoric acid from acid phosphate. Found: Phosphoric acid, soluble, 6.08; reverted, 4.16 ; insoluble, 3.51 ; total, I4.75; available, II.24.

Remark: Phosphoric acid very low.

5662. BUCKEYE. Walter Marshall, Agent, Wheeling, W. Va. Guarantee: Phosphoric acid, total, Io; available, 8; nitrogen, 2 ; potash, 3 ; phosphoric acid $3 / 4$ from bone and bone tankage, $1 / 4$ acid. Nitrogen, bone $1 / 2$ and bone tankage $1 / 2$; potash from kainit. Found: Phosphoric acid, soluble, 0.77 ; reverted, 7.47 ; insoluble, $3.6 \mathrm{I}$; total, II.85; available, 8.24 ; nitrogen, 99 ; potash, 2.72 ; availability of nitrogen, 80 ; chlorine, 3 .

Remark: Nitrogen very low.

5503. CORN, OATS AND WHEAT GROWER. Walter Marshall, Agent, Wheeling, W. Va. Guarantee: Phosphoric

\section{MUSKINGUM VALLEY FERTILIZER CO.,}

\section{MarietTTA, OHio.}

5485. PEERLESS BRAND SPECIAL VEGETABLE GROWER. West Charleston Feed Co., Agent, Charleston, W. Va. Guarantee: Phosphoric acid, soluble, 7; reverted, I.50; insoluble, I.5O; total, Io; available, 9; nitrogen, I; potash, 4; phosphoric acid from rock; nitrogen from sheep guano, 50\%, animal tankage $50 \%$, potash from sheep guano, $40 \%$, high grade muriate, 3.6\%. Found: Phosphoric acid, soluble, 5.80; reverted, 3.20 ; insoluble, I.59; total, I0.59; available, 9 ; nitrogen, I.23; potash, 3.63 ; availability of nitrogen, 60 ; chlorine, .7.

Remark: Potash low. 
5561. PEERLESS BRAND SPECIAL VEGETABLE GROWER. Johnson Implement Co., Agent, Parkersburg, W. Va. Guarantee: (As above.) Found: Phosphoric acid, soluble, 5.64; reverted, 3.I3; insoluble, I.6I ; total, Io.48; available, 8.87 ; nitrogen, I ; potash, 4.69 ; availability of nitrogen, 63 ; chlorine, I.I.

550I. PEERLESS BRAND SPECIAL VEGETABLE GROWER. Walter Marshall, Agent, Wheeling, W. Va. Guarantee: (As above.) Found: Phosphoric acid, soluble, 7.55; reverted, 2.82 ; insoluble, I.83; total, I2.20; available, I0.37; nitrogen, I.44; potash, $3.8 \mathrm{I}$; availability of nitrogen, 60 ; chlorine, I.

5560. PEERLESS BRAND ACIDULATED BONE PHOSPHATE. Johnson Implement Co., Agent, Parkersburg, W. Va. Guarantee: Phosphoric acid, soluble, 8; reverted, I.5O; insoluble, I.50; total, II.00; available, I0.00; nitrogen, I.00; potash, I.OO; phosphoric acid from rock; nitrogen from sheep guano $50 \%$, animal tankage $50 \%$, potash from sheep guano, $25 \%$ high grade muriate, $75 \%$. Found: Phosphoric acid, soluble, 3.08 ; reverted, 4.93 ; insoluble, 3.I2; total, I I.I3; available, 8.0 ; nitrogen, I.30; potash, I.85; availability of nitrogen, $6 \mathrm{I}$; chlorine, .8 .

5566. PEERLESS BRAND ACID PHOSPHATE. Johnson Implement Co., Agent, Parkersburg, W. Va. Guarantee: Phosphoric acid, soluble, IO; reverted, I.50; insoluble, I.50; total, I3; available, I2; phosphoric acid from rock. Found: Phosphoric acid, soluble, $3.5^{8}$; reverted, 4.I4; insoluble, 3.53 ; total, II.25; available, 7.72.

Remark: Available phosphoric acid very low.

5585. PEERLESS BRAND BONE MEAL. Johnson Implement Co., Agent, Parkersburg, W. Va. Guarantee: Phosphoric acid, total, 20.00; nitrogen, 2.50; phosphoric acid from bone; nitrogen from bone. Found: Phosphoric acid, total, 21.39; nitrogen, 2.99; availability of nitrogen, 92. 
D. B. MARTIN CO.,

Philadeliphia, Baltimore, AND NORFOLK.

5680. MARTIN'S SURE GROWER. Washington \& Alexander, Agent, Charles Town, W. Va. Guarantee: Phosphoric acid, soluble, 5 ; reverted, 3 ; insoluble, 4 ; total, I2; available, 8 ; nitrogen, 0.823 ; potash, $\mathrm{I}$; phosphoric acid from animal bones and slaughter house refuse; nitrogen from animal bones abattoir refuse tankage; potash from muriate of potash. Found: Phosphoric acid, soluble, 3.97 ; reverted, 6.26; insoluble, 2.78; total, I3.OI ; available, I0.23; nitrogen, I.O2; potash, I.OI ; availability of nitrogen, 89; chlorine, 2.8 .

Remark: Source of potash equivalent to kainit.

5683. MARTIN'S DISSOLVED ORGANIC COMPOUND. Washington \& Alexander, Agent, Charles Town, W. Va. Guarantee: Phosphoric acid, soluble, 5; reverted, 3; insoluble, 4; total, I2; available, 8 ; nitrogen, I.646; potash, 2; phosphoric acid from animal bones and slaughter house refuse; nitrogen from animal bone abattoir refuse tankage; potash from muriate of potash. Found: Phosphoric acid, soluble, 5.44; reverted, 5.55; insoluble, 2.20; total, I3.I9; available, I0.99; nitrogen, I.23; potash, I.69; availability of nitrogen, 9I ; chlorine, I.4.

Remark: Potash low; nitrogen low.

5676. PURE GROUND BONE. Washington \& Alexander, Agent, Charles Town, W. Va. Found: Phosphoric acid, total, 24.22 ; nitrogen, 2.34; availability of nitrogen, 94.

5675. MARTIN'S ANIMAL 6\% TANKAGE. Washington \& Alexander, Agent, Charles Town, W. Va. Guarantee: Phosphoric acid, total, I3.74; nitrogen, 4.938; phosphoric acid from animal bones; nitrogen from animal bone, animal meat, animal offal. Found: Phosphoric acid, total, 7.96; nitrogen, 5.92 ; availability of nitrogen, 93 .

Remark: Total phosphoric acid low. 


\section{OHIO FARMERS FERTILIZER CO.,}

\section{Columbus, Ohio.}

5496. POTATO AND TOBACCO SPECIAL. Stortz, Blazer Co., Agent, Point Pleasant, W. Va. Guarantee: Phosphoric acid, soluble, 5 ; reverted, 3 ; total, Io; available, 8 ; nitrogen, r.64; potash, 4 ; phosphoric acid from dissolved bone phosphate; nitrogen, two-fifths to three-fifths from bone tankage, two-fifths to three-fifths from blood, one-fifth to two-fifths from tobacco-stems; potash three-fourths to four-fifths from muriate of potash, one-fourth to one-half from tobacco stems. Found: Phosphoric acid, soluble, 2.56; reverted, 6.02; insoluble, 2.88; total, Ir.46; available, 8.58 ; nitrogen, I.49; potash, 4.21 ; availability of nitrogen, 79 ; chlorine, I.

Remark: Nitrogen low.

5639. POTATO AND TOBACCO SPECIAL. W. P. Mason \& Co., Agent, Mannington, W. Va. Guarantee: (As above.) Found: Phosphoric acid, soluble, .54; reverted, 6.44; insoluble, 2.89; total, 9.87 ; available, 6.98; nitroger, .97; potash, 4.I I ; availability of nitrogen, 69; chlorine, 2.2.

Remark: Nitrogen low. Source of potash equivalent to manure salts.

5497. GENERAL CROP FISH GUANO. Stortz, Blazer \& Co., Pt. Pleasant, W. Va. Guarantee: Phosphoric acid, soluble, 4 ; reverted, 3 ; total, 9 ; available, 7 ; nitrogen, 0.82 ; potash, I ; phosphoric acid from dissolved phosphate rock; nitrogen, $1 / 4$ to $1 / 2$ from bone tankage, $1 / 4$ to $1 / 2$ from blood, $1 / 4$ to $1 / 2$ from tobacco stems, $I / 4$ to $1 / 2$ from fish scrap; potash, $1 / 2$ to $3 / 4$ from muriate potash, $1 / 2$ to $3 / 4$ from tobacco stems. Found: Phosphoric acid, soluble, 2.06; reverted, 5.9r ; insoluble, 3.08; total, I 1.05; available, 7.97 ; nitrogen, 0.77 ; potash, 3.83 ; availability of nitrogen, 78 ; chlorine, .9. 


\section{G. OBER \& SONS CO.,}

BALTIMORE, MD.

5748. OBER'S FARMERS MIXTURE. A. G. Chrislip, Agent, Philippi, W. Va. Guarantee: Phosphoric acid, soluble, 7 ; reverted, 2 ; insoluble, 2 ; total, I I ; available, 9 ; nitrogen, 0.82 ; potash, 2; phosphoric acid from high grade Florida phosphate, eight-ninths bone from tankage and fish, one-ninth nitrogn, $\mathrm{H}$. G. Bone and blood tankage, $1 / 2$ to $3 / 4$, fish, $1 / 2$ to $3 / 4$; potash from sulphate of potash. Found: Phosphoric acid, soluble, 7.II; reverted, I.73; insoluble, 2.33 ; total, II.I7; available, 8.84 ; nitrogen, 0.83 ; potash, 2.66 ; availability of nitrogen, 87 ; chlorine, 2.4 .

Remark: Source of potash equivalent to manure salts.

5749. OBER'S DISSOLVED BONE PHOSPHATE. A. G. Chrislip, Agent, Philippi, W. Va. Guarantee: Phosphoric acid, soluble, II ; reverted, 3 ; insoluble, 2 ; total, I6; available, I4; phosphoric acid from H. G. Florida phosphate. Found: Phosphoric acid, soluble, I3.37; reverted, 2.03; insoluble, 0.27 ; total, I5.67; available, I 5.40.

5547. OBER'S DISSOLVED BONE PHOSPHATE. R. N. Stewart \& Son, Agent, Martinsburg, W. Va. Guarantee: (As above.) Found: Phosphoric acid, soluble, I3.98; reverted, 2.25 ; insoluble, 0.46; total, I6.69; available, I6.23.

568I. OBER'S DISSOLVED BONE PHOSPHATE. Washington \& Alexander, Agent, Charles Town, W. Va. Guarantee: (As above.) Found: Phosphoric acid, soluble, I4.OI; reverted, 2.43; insoluble, .09; total, I6.53; available, I6.44.

5684. OBER'S DISSOLVED BONE PHOSPHATE. R. N. Stewart \& Son, Agent, Martinsburg, W. Va. Guarantee: (As above.) Found: Phosphoric acid, soluble, I4.09; reverted, 3.09; insoluble, 0.27 ; total, 17.45; available, I7.18. 


\section{PIEDMONT MT. AIRY GUANO CO.,}

BALTIMORE, MD.

5540. PIEDMONT FARMER'S FAVORITE. Cicero Phillips, Agent, Belington, W. Va. Guarantee: Phosphoric acid, insoluble, 2 ; total, Io ; available, 8 ; nitrogen, 0.82 ; potash, 4; phosphoric acid from dissolved phosphate rock and dissolved bone tankage; nitrogen $1 / 2$ blood and $1 / 2$ bone tankage; potash $1 / 2$ muriate of potash and $1 / 2$ kainit. Found: Phosphoric acid, soluble, 7.22 ; reverted, I.68; insoluble, I.OI ; total, 9.9I ; available, 8.90 ; nitrogen, 0.66 ; potash, 2.86 ; availability of nitrogen, 57 ; chlorine, 3.6.

Remark: Nitrogen low. Potash low. Source of potash equivalent to kainit. Chlorine excessive.

5753. PIEDMONT FARMER'S FAVORITE. G. W. Neiswander, Agent, Parkersburg, W. Va. Guarantee: (As above.) Found: Phosphoric acid, soluble, 5.32; reverted, 2.13; insoluble, 0.72 ; total, 8.17 ; available, 7.45 ; nitrogen, 0.78 ; potash, 4.42 ; availability of nitrogen, 60 ; chlorine, 2.5 .

Remark: Available phosphoric acid low.

\section{I. PIEDMONT SPECIAL POTASH MIXTURE.} Ci_ero Phillips, Agent, Belington, W. Va. Guarantee: Phosphoric acid, insoluble, I ; total, II ; available, Io; potash, 5 ; phosphoric acid from dissolved phosphate rock; potash, $1 / 2$ from muriate of potash, I/2 from kainit. Found: Phosphoric acid, soluble, 5.28 ; reverted, 4.60 ; insoluble, 0.43 ; total, I0.3I ; available, 9.88 ; potash, 4.80 ; chlorine; r.9.

5542. PIEDMONT GENERAL CROP GROWER. Cicero Phillips, Agent, Belington, W. Va. Guarantee: Phosphoric acid, available, 8 ; nitrogen, .82 ; potash, I ; phosphoric acid from bone phosphate; nitrogen, $1 / 2$ from bone tankage, $1 / 2$ from blood; potash, $1 / 2$ kainit; $1 / 2$ muriate. Found: Phosphoric acid, soluble, 6.50; reverted, 2.55 ; insoluble; I. 30 ; total, 10.35; available, 9.05 ; 
nitrogen, 0.70; potash, I.07; availability of nitrogen, 62; chlorine, 3 .

Remark: Nitrogen low. Source of potash equivalent to kainit.

5543. PIEDMONT FARMER'S HIGH GRADE BONE AND POTASH. Cicero Phillips, Agent, Belington, W. Va. Guarantee: Phosphoric acid, insoluble, 2; total, I2; available, IO ; potash, 2 ; phosphoric acid from S. C. Rock; potash from kainit. Found: Phosphoric acid, soluble, 7.85; reverted, 2.76; insoluble, 0.46 ; total, I I.07; available, I0.61 ; potash, I.84; chlorine, 3.7 .

Remark: Source of potash equivalent to kainit; chlorine, excessive.

5750. PIEDMONT POTATO PRODUCER. G. W. Neiswander, Agent, Parkersburg, W. Va. Guarantee: Phosphoric acid, insoluble, 2 ; total, 7 ; available, 5 ; nitrogen, 2.09 ; potash, 6; phosphoric acid from dissolved bone and phosphate rock; nitrogen, one-third tankage, one-third nitrate of soda and onethird blood; potash from high grade muriate of potash. Found: Phosphoric acid, soluble, 3.84; reverted, 2.29; insoluble, 0.27 ; total, 6.40; available, 6.r3; nitrogen, I.22; potash, 5.20; availa* bility of nitrogen, 78 ; chlorine, 1.3 .

Remark: Nitrogen low; potash low; chlorine high.

575I. PIEDMONT PURE RAW BONE MIXTURE. G. W. Neiswander \& Co., Agent, Parkersburg, W. Va. Guarantee: Phosphoric acid, insoluble, 4; total, I2; available, 8; nitrogen, 1.02; potash, 2; phosphoric acid from dissolved bone tankage; nitrogen from bone tankage; potash $1 / 2$ from muriate, I/2 from kainit. Found: Phosphoric acid, soluble, 5.56; reverted, 4.84 ; insoluble, 2.12; total, 12.52 ; available, I0.40; nitrogen, I.44; potash, 2.07 ; availability of nitrogen, 90 ; chlorine, 3.2 .

Remark: Source of potash equivalent to kainit. Chlorine excessive.

5752. LEVERING'S EXCELSIOR. G. W. Neiswander \& 
Co., Agent, Parkersburg, W. Va. Guarantee: Phosphoric acid, insoluble, I ; total, 9; available, 8; nitrogen, 0.82 ; potash, I; phosphoric acid from dissolved phosphate rock and dissolved bone tankage; nitrogen, $1 / 2$ blood, and $1 / 2$ bone tankage; potash $1 / 2$ from muriate of potash, $\mathrm{T} / 2$ from kainit. Found: Phosphoric acid, soluble, 3.56 ; reverted, 3.63 ; insoluble, 1.16; total, 8.35; available, 7.19; nitrogen, I.04; potash, I.IO; availability of nitrogen, 53; chlorine, 2.4 .

Remark: Source of potash equivalent to kainit. Available phosphoric acid low.

5689. PIEDMONT HIGH GRADE S. C. BONE. C. W. Mayers \& Son, Terra Alta, W. Va. Guarantee: Phosphoric acid, insoluble, I ; total, 15; available, 14; phosphoric acid from dissolved S. C. Rock. Found: Phosphoric acid, soluble, II.58; reverted, 3.43 ; insoluble, 0.28; total, I5.29; available, I5.0I.

\section{RASIN MONUMENTAL CO., \\ P. O. Box 97I. \\ BALTIMORE, MD.}

5670. RASIN'S BONE AND POTASH FERTILIZER. Washington \& Alexander, Agent, Charles Town, W. Va. Guarantee: Phosphoric acid, soluble, 8; reverted, 2; insoluble, $\mathrm{I}$; total, II ; available, Io; potash, 2; phosphoric acid from high grade Florida and Tennessee phosphate; potash $1 / 4$ to $1 / 2$ high grade manure salts, $1 / 2$ to $3 / 4$ kainit. Found: Phosphoric acid, soluble, 4.09; reverted, 5.37 ; insoluble, 2.25 ; total, Ir.7I ; available, 9.46; potash, 2.37 ; chlorine, 4 .

Remark: Source of potash equivalent to kainit. Chlorine excessive. 
5678. RASIN'S ACID PHOSPHATE. Washington \& Alexander, Agent, Charles Town, W. Va. Guarantee: Phosphoric acid, soluble, I2; reverted, 2 ; insoluble, I ; total, I5 ; available, I4; phosphoric acid from high grade Florida and Tennessee phosphate. Found: Phosphoric acid, soluble, 8.37 ; reverted, 7.48; insoluble, I.56; total, I7.4I ; available, I 5.85 .

5706. RASIN'S ACID PHOSPHATE. George Carskaddon, Agent, Keyser, W. Va. Guarantee: (As above.) Found: Phosphoric acid, soluble, Ir.8I; reverted, 4.66; insoluble, 0.97 ; total, 17.44 ; available, 16.57 .

5674. WASHINGTON \& ALEXANDER'S SPECIAL MIXTURE FOR WHEAT. Washington \& Alexander, Agent, Charles Town, W. Va. Guarantee: Phosphoric acid, soluble, 7 ; reverted, 2 ; insoluble, r.50; total, 10.50; available, 9; nitrogen, 0.82 ; potash, 2; phosphoric acid from high grade Florida and Tennessee phosphate; nitrogen, $1 / 4$ to one-third high grade fish, one-third to $1 / 2$ high grade tankage, $1 / 4$ to one-third blood; potash, $1 / 4$ to one-third high grade muriate of potash, two-thirds to $3 / 4$ high grade manure salts. Found: Phosphoric acid, soluble, 5.69; reverted, 3.27 ; insoluble, 2.29; total, II.25; available, 8.96; nitrogen, I; potash, 3.37 ; availability of nitrogen, 77 ; chlorine, 3.4 .

Remark: Source of potash equivalent to kainit. Chlorine excessive.

\section{SCIENTIFIC FERTILIZER CO.,}

Pit'Tsburg, Pa.

5502. PURE RAW BONE MEAL. Walter Marshall, Agent, Wheeling, W. Va. Found: Phosphoric acid, total, 20.20 ; nitrogen, 3.61 ; availability of nitrogen, 85 . 


\section{SWIFT \& CO.,}

Chicago, Illi.

5474. SWIFT'S SUPERPHOSPHATE. J. M. Miller \& Bro., Agent, Ronceverte, W. Va. Guarantee: Phosphoric acid, insoluble, 4; total, I2 ; available, 8 ; nitrogen, I.64; potash, 2 ; phosphoric acid about one-third bone and two-thirds acid phosphate; nitrogen, two-thirds bone and one-third meat; potash from muriate. Found: Phosphoric acid, soluble, 4.38; reverted, 3.67 ; insoluble, I.74; total, 9.79; available, 8.05 ; nitrogen, I.52; potash, I.90; availability of nitrogen, 90; chlorine, 0.03 .

Remark: Nitrogen low.

5654. SWIFT'S SUPERPHOSPHATE. T. P. Licklider, Agent, Martinsburg, W. Va. Guarantee: (As above.) Found: Phosphoric acid, soluble, 4.I8; reverted, 4.74; insoluble, I.38; total, I0.30 ; available, 8.92 ; nitrogen, I.5I ; potash, I.92 ; availability of nitrogen, $9 \mathrm{I}$; chlorine, .8 .

Remark: Nitrogen low.

5677. SWIFT'S SUPERPHOSPHATE. Washington \& Alexander, Agent, Charles Town, W. Va. Guarantee: (As above.) Found: Phosphoric acid, soluble, 3.26; reverted, 6.39; insoluble, 2.I6; total, II.8I ; available, 9.65; nitrogen, I.85; potash,-2.06; availability of nitrogen, 93 ; chlorine, .7.

5475. PURE BONE MEAL. J. M. Miller \& Bro., Agent, Ronceverte, W. Va. Guarantee: Phosphoric acid, total, 25; nitrogen, 2.50; phosphoric acid from bone; nitrogen, from bone. Found: Phosphoric acid, total, 24.92; nitrogen, 2.34 ; availability of nitrogen, 87 .

Remark: Nitrogen low.

5704. PURE BONE MEAL. George Carskaddon, Agent, Keyser, W. Va. Guarantee: (As above.) Found: Phosphoric acid, total, 26.33; nitrogen, 2.25; availability of nitrogen, 90.

Remark: Nitrogen low. 
5754. PURE BONE MEAL. G. T. Hodges, Agent, Shepherdstown, W. Va. Guarantee: (As above.) Found: Phosphoric acid, total, 25.92; nitrogen, 2.50; availability of nitrogen, 9I.

5476. COMPLETE FERTILIZER. J. M. Miller \& Bro., Agent, Ronceverte, W. Va. Guarantee: Phosphoric acid, insoluble, 3 ; total, II ; available, 8 ; nitrogen, I ; potash, I ; phosphoric acid about one-third bone and two-thirds acid phosphate; nitrogen, two-thirds bone, one-third meat; potash from muriate. Found: Phosphoric acid, soluble, 4.9I ; reverted, 4.87 ; insoluble, I.84; total, I1.62; available, 9.78 ; nitrogen, 1.26; potash, I.45; availability of nitrogen, 85 ; chlorine, 0.4 .

5660. COMPLETE FERTILIZER. T. P. Licklider, Agent, Martinsburg, W. Va. Guarantee: (As above.) Found: Phosphoric acid, soluble, 3.24 ; reverted, 5.75 ; insoluble, I.73; total, I0.72; available, 8.99; nitrogen, 0.99; potash, 0.97 ; availability of nitrogen, 88 ; chlorine, .8 .

5755. COMPLETE FERTILIZER. G. T. Hodges, Agent, Shepherdstown, W. Va. Guarantee: (As above.) Fiound: Phosphoric acid, soluble, 3.52; reverted, 5.59; insoluble, 2.01; total, II.I2; available, 9.II ; nitrogen, I.29; potash, I.I2; availability of nitrogen, 90 ; chlorine, .9.

5480. PURE RAW BONE MEAL. J. M. Miller \& Bro., Agent, Ronceverte, W. Va. Guarantee: Phosphoric acid, total, 23; nitrogen, 3.75; phosphoric acid from bone; nitrogen from bone. Found: Phosphoric acid; total, 21.86; nitrogen, 3.95; availability of nitrogen, 82 .

Remark: Phosphoric acid low.

5659. CHAMPION WHEAT AND CORN GROWER. T. P. Licklider, Agent, Martinsburg, W. Va. Guarantee: Phosphoric acid, insoluble, I; total, I3; available, I2; nitrogen, I.64; potash, 2; phosphoric acid from bone; nitrogen from bone; potash from muriate. Found: Phosphoric acid, soluble, I.28; 
reverted, 0.58; insoluble, I.I6; total, I3.02 ; available, II.86; nitrogen, I.70; potash, 2.I8; availability of nitrogen, 91 ; chlorine, .6.

\section{P. THOMAS \& SON CO., \\ 2 S. Deliaware Avenue, Philadeliphia, Pa.}

5655. S. C. PHOSPHATE. T. P. Licklider, Agent, Martinsburg, W. Va. Guarantee: Insoluble, I ; total, I5; available, I4; phosphoric acid from phosphate rock. Found: Phosphoric acid, soluble, I2.79; reverted, 3.32 ; insoluble, 0.32; total, I6.43; available, I6.II.

5656. ALKALINE BONE. T. P. Licklider, Agent, Martinsburg, W. Va. Guarantee: Insoluble, I; available, ro; potash, 2; phosphoric acid from phosphate rock; potash from kainit. Found: Phosphoric acid, soluble, 2.I7; reverted, 8.I3; insoluble, I.o8; total, II.39; available, I0.30; pctash, 2.37; chlorine, I.6.

\section{TUSCARORA FERTILIZER CO.,}

BALTIMORE, MD.

57IO. CEREAL PHOSPHATE. Parker, Smith \& Ours, Agent, Petersburg, W. Va. Guarantee: Phosphoric acid, soluble, 8 ; reverted, 2 ; insoluble, 2 ; total, I2 ; available, Io; phosphoric acid from acid phosphate. Found: Phosphoric acid, soluble, 5.56; reverted, 3.29 ; insoluble, I.O7; total, IO.32 ; available, 9.25 .

Remark: Available phosphoric acid low.

5738. ACID PHOSPHATE. Philippi Mill Co., Agent, 
Philippi, W. Va. Guarantee: Phosphoric acid, soluble, I2; reverted, 2 ; insoluble, 2 ; total, I6; available, I4; phosphoric acid from acid phosphate. Found: Phosphoric acid, soluble, I3.05; reverted, 2.98; insoluble, 0.78 ; total, I6.8I ; available, I6.03.

5773. ACID PHOSPHATE. R. Hunter, Agent, Berkeley Springs, W. Va. Guarantee: (As above.) .Found: Phosphoric acid, soluble, II.I3; reverted, 4.80; insoluble, .79; total, I6.72 ; available, I 5.93.

5564. ACID PHOSPHATE. J. H. Manear \& Son, Martinsburg, W. Va. Guarantee: (As above.) Found: Phosphoric acid, soluble, 9.6I ; reverted, 4.I2; insoluble, I.06; total, I4.79; available, I3.73.

Remark: Available phosphoric acid low.

5739. BONE AND POTASH. Philippi Mill Co., Agent, Philippi, W. Va. Guarantee: Phosphoric acid, soluble, 8; reverted, 2 ; insoluble, 2 ; total, I2 ; available, Io ; potash, 2 ; phosphoric acid from acid phosphate; potash three-fifths to fourfifths from kainit, one-fifth to two-fifths from muriate of potash. Found: Phosphoric acid, soluble, 7.87; reverted, 2.80 ; insoluble, I.07 ; total, I I.74; available, I0.67; potash, 2.26 ; chlorine, 3.3 .

Remark: Source of potash equivalent to kainit; chlorine excessive.

577I. BONE AND POTASH. R. Hunter, Agent, Berkeley Springs, W. Va. Guarantee: (As above.) Found: Phosphoric acid, soluble, 6.52; reverted, 3.84 ; insoluble, 0.46 ; total, I0.82; available, I0.36; potash, I.92; chlorine, 4.4 .

Remark: Source of potash equivalent to kainit: Chlorine excessive. 


\title{
VIRGINIA-CAROLINA CHEMICAL, CO.,
}

\author{
RICHMOND, VA.
}

5548. V. C. C. CO'S. DISSOLVED BONE. T. P. Licklider, Agent, Martinsburg. Found: Phosphoric acid, soluble, 5.8I; reverted, 5.38; insoluble, 4.38; total, I5.57; available, Ir.I9; nitrogen, 2.08; availability of nitrogen, 90 .

5716. V. C. C. CO'S. SPECIAL POTASH MIXTURE. J. M. Miller \& Bro., Agent, Ronceverte, W Va. Guarantee: Phosphoric acid, soluble, 7 ; reverted, 3 ; insoluble, I ; total, "II ; available, Io; potash, 4 ; phosphoric acid from phosphate rock; potash, $\mathrm{T} / 2$ from muriate, $\mathrm{T} / 2$ from double manure salts. Found: Phosphoric acid, soluble, 3.86; reverted, 5.07; insoluble, I.6I; total, Io.54; available, 8.93 ; potash, 5.85 ; chlorine, I.4.

Remark: Available phosphoric acid low.

5483. ALLISON \& ADDISON'S LITTLE GIANT GRAIN AND GRASS GROWER. J. M. Miller \& Bro., Agent, Ronceverte, W. Va. Guarantee: Soluble, 6; reverted, 2; insoluble, 2 ; total, Io; available, 8 ; nitrogen, 0.82 ; potash, 2 ; phosphoric acid four-fifths from phosphate rock, one-fifth animal bone; nitrogen, $\mathrm{T} / 4$ from fish, $\mathrm{x} / 4$ from blood, $\mathrm{x} / 4$ from animal tankage, $1 / 4$ from nitrate of soda; potash, $1 / 2$ from muriate and $1 / 2$ from double manure salts or kainit. Found: Phosphoric acid, soluble, 3.6I ; reverted, 3.40; insoluble, 4.I8; total, II.19; available, 7.01 ; nitrogen, 0.9I ; potash, 2.19 ; availability of nitroegn, 78 ; chlorine, I.2.

Remark: Phosphoric acid low.

553I. ALLISON \& ADDISON'S LIT'TLE GIANT GRAIN AND GRASS GROWER. E. Shoemaker, Agent, Marlinton, W. Va. Guarantee: (As above.) Found: Phosphoric acid, soluble, 4.30; reverted, 4.24; insoluble, I.36; total, 9.90; available, 8.54 ; nitrogen, 0.90 ; potash, I. $6 \mathrm{I}$; availability of nitrogen, 79; chlorine, 2.7.

Remark: Potash low. Source of potash equivalent to kainit. 
57I2. ALLISON \& ADDISON'S LITTLE GIANT GRAIN AND GRASS GROWER. J. M. Miller \& Bro., Agent, Ronceverte, W. Va. Guarantee: (As above.) Found: Phosphoric acid, soluble; 6.3I ; reverted, 2.66; insoluble, .77; total, 9.74; available, 8.97 ; nitrogen, .94; potash, I.76; availability of nitrogen, 93 ; chlorine, 2.5 .

Remark: Source of potash equivalent to kainit.

5484. ALLISON \& ADDISON'S B. P. POTASH MIXTURE. J. M. Miller \& Bro., Agent, Ronceverte, W. Va. Guarantee: Phosphoric acid, soluble, 7 ; reverted, 3 ; insoluble, I ; total, II ; available, Io; potash, 2 ; phosphoric acid from phosphate rock; potash, $1 / 2$ from muriate, $1 / 2$ from double manure salts or kainit. Found: Phosphoric acid, soluble, 4.06; reverted, 5.40; insoluble, I.4I ; total, I0.87; available, 9.46; potash, 2.55; chlorine, I.5.

Remark: Available phosphoric acid low.

5532. ALLISON \& ADDISON'S STANDARD ACID PHOSPHATE. E Shoemaker, Agent, Marlinton, W. Va. - Guarantee: Phosphoric acid, soluble, 9; reverted, 3; insoluble, I ; total, I3; available, I2; phosphoric acid from phosphate rock. Found: Phosphoric acid, soluble, 6.90; reverted, 6.60; insoluble, I.24; total, I4.74; available, I3.50.

5482. SOUTHERN CHEMICAL COMPANY'S SUN $B R A N D$ GUANO. J. M. Miller \& Bro., Agent, Ronceverte, W. Va. Guarantee: Phosphoric acid, soluble, 6; reverted, 2 ; insoluble, 2 ; total, Io ; available, 8 ; nitrogen, 2.06 ; potash, 5 ; phosphoric acid, four-fifths phosphate rock, one-fifth animal bone; nitrogen, $I / 4$ from fish, $I / 4$ from blood, and $1 / 4$ from animal tankage, $1 / 4$ from nitrate of soda; potash, $1 / 2$ from muriate, $1 / 2$ double manure salts or kainit. Found: Phosphoric acid, soluble, 4.80; reverted, 2.52 ; insoluble, 0.92 ; total, 8.24 ; available, 7.32 ; nitrogen, 2.IO; potash, 4.88; availability of nitrogen, 90 ; chlorine, .I. Remark: Phosphoric acid low.

5529. SOUTHERN CHEMICAL CO'S SUN BRAND 
GUANO. E. Shoemaker, Agent, Marlinton, W. Va. Guarantee: (As above.) Found: Phosphoric acid, soluble, 5.58; reverted, 3.75 ; insoluble, 0.99 ; total, 9.22 ; available, 8.23 ; nitrogen, 2.13; potash, 4.63 ; availability of nitrogen, 91 ; chlorine, I.3.

57I4. S. W. TRAVERS \& CO'S. CAPITAL BONE AND POTASH COMPOUND. J. M. Miller \& Bro., Agent, Ronceverte, W. Va. Guarantee: Phosphoric acid, soluble, 7 ; reverted, 3 ; insoluble, I ; total, II ; available, IO; potash, 2 ; phosphoric acid from phosphate rock; potash $1 / 2$ from muriate, $1 / 2$ from double manure salts or kainit. Found: Phosphoric acid, soluble, 3.58; reverted, 6.56; insoluble, I.62; total, I I.76; available, IO.I4; potash, I.83; chlorine, 2.I.

5762. CASLËR'S WHEAT AND GRASS COMPOUND. C. E. Casler, Agent, Berkeley Springs, W. Va. Guarantee: Phosphoric acid, soluble, 4; reverted, 2; insoluble, I; total, 7; available, 6; potash, 3; phosphoric acid from high grade Florida Tennessee and Charleston phosphate; potash, $1 / 4$ to $1 / 2$ high grade manure salts, $1 / 4$ to $1 / 2$ muriate of potash, $1 / 4$ to $1 / 2$ kainit. Found: Phosphoric acid, soluble, 0.95; reverted, 6.27; insoluble, 0.97 ; total, 8.19; available, 7.22; potash, 3.87 ; chlorine, 3.5 .

Remark: Source of potash equivalent to kainit. Chlorine excessive.

\section{ROBERT A. WOOLDRIDGE CO.,}

BalTIMORE, MD.

5460. OLD SLEDGE POTASH MIXTURE. J. D. Summers, Agent, Little Falls, W. Va. Guarantee: Phosphoric acid, soluble, Io; reverted, 2 ; insoluble, I ; available, I2; potash, 5; phosphoric acid from dissolved phosphate rock; potash, twofifths from muriate of potash, three-fifths from manure salt. Found: Phosphoric acid, soluble, 9.25; reverted, 2.98; insoluble, 0.4I ; total, I2.63; available, I2.23; potash, 3.69; chlorine, I.6.

Remark: Potash low. 
5463. OJD SLEDGE POTASH MIXTURE. J. D. Summers, Agent, Little Falls, W. Va. Guarantee: (As above.) Found: Phosphoric acid, soluble, 9.42 ; reverted, 2.80 ; insoluble, 0.56 ; total, I2.78; available, I2.22 ; potash, 5.25 ; chlorine, I.3.

546I. LIBERTY BELL POTASH MIXTURE. J. D. Summers, Agent, Little Falls, W. Va. Guarantec: Phosphoric acid, soluble, Io; reverted, 2; insoluble, I; available, I2; potash, 3 ; phosphoric acid from dissolved phosphate rock; potash, $1 / 2$ from manure salt, $1 / 2$ from muriate. Found: Phosphoric acid, soluble, 9.30 ; reverted, 2.57 ; insoluble, 0.4I ; total, I2.28; available, II.97; potash, 2.96; chlorine, 2.

Remark: Source of potash equivalent to manure salt.

5462. CHAMPION GIANT PHOSPHATE. J. D. Summers, Agent, Little Falls, W. Va. Guarantee: Phosphoric acid, soluble, 7 ; reverted, 2 ; insoluble, 1.50 ; available, 9; nitrogen, 0.82 ; potash, 2; phosphoric acid $3 / 4$ from dissolved phosphate rock, $1 / 4$ from Am. bone in tankage; nitrogen, two-fifths fish, three-fifths high grade tankage; potash, from kainit. Found: Phosphoric acid, soluble, 6.40; reverted, 2.74; insoluble, I.39; total, I0.53; available, 9.14; nitrogen, o.86; potash, 2.13 ; availability of nitrogen, 75 ; chlorine, I.9.

5699. CHAMPION GIANT PHOSPHATE. I. J. Lohr, Agent, Belington, W. Va. Guarantee: (As above.) Found: Phosphoric acid, soluble, 5.56; reverted, 2.40; insoluble, I.25; total, 9.21 ; available, 7.96 ; nitrogen, 0.85 ; potash, 2.03 ; availability of nitrogen, 73 ; chlorine, 2.5 .

Remark: Phosphoric acid low.

5464. GERMAN POTASH MIXTURE. J. D. Summers, Agent, Little Falls, W. Va. Guarantee: Phosphoric acid, soluble, 8 ; reverted, 2 ; insoluble, I ; available, Io ; potash, 2 ; phosphoric acid from dissolved phosphate rock; potash from kainit. Found: Phosphoric acid, soluble, 6.88; reverted, 3.08 ; insoluble. o.86; total, Io.82; available, 9.96; potash, 2.08 ; chlorine, $3 . \mathrm{I}$

Remark: Chlorine excessive. 
5698. GERMAN POTASH MIXTURE. I. J. Lohr, Agent, Belington, W. Va. Guarantee: (As above.) Found: Phosphoric acid, soluble, 6.9I ; reverted, 3.30; insoluble, 0.4I ; total, I0.62; available, I0.2I ; potash, 2.19; chlorine, 3.2.

Remark: Chlorine excessive.

5470. SPECIAL, POTATO FERTILIZER. J. D. Summers, Agent, Little Falls, W. Va. Guarantee: Phosphoric acid, soluble, 7 ; reverted, 2 ; insoluble, 1.50; available, 9; nitrogen, I.64; potash, 5 ; phosphoric acid, $3 / 4$ from dissolved phosphate rock, $1 / 4$ bone in tankage; nitrogen, two-fifths from fish, threefifths from tankage; potash, $1 / 2$ from muriate, $1 / 2$ from high grade manure salts. Found: Phosphoric acid, soluble, 6.79; reverted, 2.58 ; insoluble, I.54; total, IO.9I ; available, 9.37 ; nitrogen, I.78; potash, 4.46; availability of nitrogen, 84 ; chlorine, I.

5697. FLORIDA ACID PHOSPHATE. I. J. Lohr, Agent, Belington, W. Va. Guarantee: Phosphoric acid, soluble, I2; reverted, 2; available, I4; phosphoric acid from dissolved phosphate rock. Found: Phosphoric acid, soluble, II.32; reverted, 4.01 ; insoluble, 0.6r ; total, I5.94; available, I 5.33.

\section{THE WOOLDRIDGE ORCHILIAA CO.,}

BALTIMORE, MD.

5685. ORCHILLA GUANO. Parley DeBarry, Agent, Terra Alta, W. Va. Guarantee: Phosphoric acid, soluble, 4; insoluble, I3; total, I7; available, 4; phosphoric acid from natural bird deposit. Found: Phosphoric acid, soluble, a trace; reverted, I0.45; insoluble, I0.49; total, 20.94; available, ro.45.

552r. BIZZY BEE. A. G. Chrislip, Agent, Philippi, W. Va. Guarantee: Phosphoric acid, soluble, 5 ; reverted, 2 ; insoluble, I.50; total, 8.50 ; available, 7 ; nitrogen, .83; potash, $\mathrm{I}$; phos- 
phoric acid, $1 / 4$ from dissolved bone tankage and $3 / 4$ from acid phosphate; nitrogen, $1 / 4$ from nitrate of soda and $3 / 4$ from dissolved bone tankage; potash from kainit. Found: Phosphoric acid, soluble, 5.4I ; reverted, 2.02; insoluble, 0.50; total, 7.93; available, 7.43 ; nitrogen, 0.79 ; potash, I.24; availability of nitrogen, 74 ; chlorine, 3.7 .

Remark: Chlorine excessive.

\section{WALKER-STRATMAN \& CO., PițTsBurg, Pa.}

5564. FOUR FOLD. W. S. Stout, Agent, Harrisville, W. Va. Found: Phosphoric acid, soluble, 5.85; reverted, 3.04 ; insoluble, I.96; reverted, 3.04 ; total, Io.85; available, 8.89 ; nitrogen, I.88; potash, I.68; availability of nitrogen, 90 ; chlorine, .7.

5450. MEAT, BLOOD AND BONE WITH POTASH. Submitted for analysis by John A. Madden, Shinnston, W. Va. Guarantee: Phosphoric acid, soluble, 6.46; reverted, 2.44 ; insoluble, 0.75 ; total, 9.65 ; available, 8.90 ; nitrogen, 2.69 ; potash, 8.54; availability of nitrogen, 96 ; chlorine, .7 .

545I. POTATO SPECIAL. Submitted for analysis by John A. Madden, Shinnston, W. Va. Found: Phosphoric acid, soluble, 5.08; reverted, 4.53; insoluble, 2.58; total, I2.I9; available, 9.6I ; nitrogen, I.79; potash, 6.59; availability of nitrogen, 9I ; chlorine, 0.7 . 
THE FOLLOWING IS A LIST OF AGENTS WHOSE, STOCK WAS SAMPLED DURING 1906.

Aldridge, W. A. ......................... Seebert Anderson, J. D. ...................... Ronceverte Braunlich, C. F. \& Co. .................... Wheeling Baylor, C. E. ....................... Charles Town Belington Ice \& Bottling Co. ............... Belington Buckhannon Grocery Co. ................. Buckhannon Carmen, C. R. ........................Wellsburg Carskadon, Geo. .......................... Keyser Casler, C. E. ..................... Berkeley Springs Chrislip, A. G. ...................... Philippi Conaway \& Clayton .................... Barracksville Corrothers, W. S. ...................... Catawba Curry \& Rogers ........................ Marlinton Damaron Seed \& Feed Co. ................ Huntington Dean \& Reeves ........................ Fairmont DeBarry, Parley ....................... Terra Alta Drummond, T. P. \& Co. ................. Buckhannon Dudley, W. R. ........................ Wheeling England, Draper ...................... Belington Exchange Mill Co. ....................... Grafton

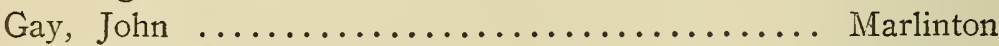
Harbor, T. M. ........................ Hurricane Hedrick, J. W. ........................ Alderson Hodges, G. T. ..................... Shepherdstown Hunter, R. .................... Berkeley Springs Jacobs, Benson ...................... Little Falls Johnson \& Gwinn .............................. Alderson Johnson Implement Co. ................. Parkersburg Lemon, W. N. \& Son .................. Shepherdstown Licklider, T. P. ..................... Martinsburg Lohr, I. J. ....................... Belington Lowndes, R. T. ...................... Clarksburg 
Marshall, Walter

Wheeling Mason, W. P. \& Co. Mannington Mayers, C. W. \& Son Terra Alta Marlinton Supply Co. Marlinton Mossman Bros. Huntington Miller, J. H. \& Son Martinsburg Miller, J. M. \& Bro. Ronceverte Myers, Orie Clarksburg Mynes Bros. Hurricane Neiswander, G. W. \& Co. ............... Parkersburg

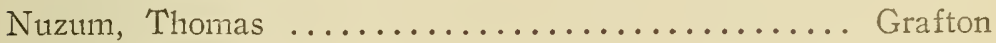
Parker, Snith \& Ours .................. Petersburg

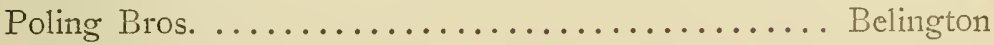
Philippi Mill Co. ................... Philippi

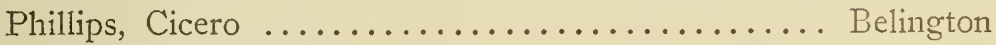
Phillips, J. H. \& Sons .............. Berkeley Springs Russell, A. P. \& Co. ................... Buckhannon Rodgers, J. M. ..................... Ronceverte

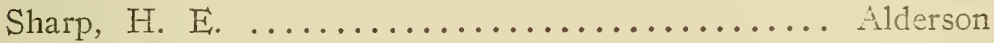
Sill, W. F. ..................... Pennsboro Seiver Hardware Co. .................... Keyser Summers, J. D. ................. Little Falls Stortz, Blazer \& Co. ................ Point Pleasant Sovine, R. W.

Hurricane Stewart, R. N. Martinsburg Shoemaker, E. Marlinton Simpson, F. A. Philippi Stout, W. S.

Harrisburg

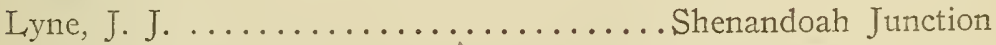
Upshur Grocery Co. .................. Buckhannon Unger, R. R. .................. Berkeley Springs Washington \& Alexander ......................... Town

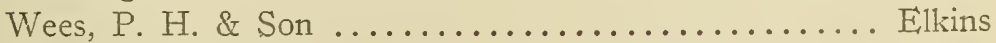
W. Va. Implement Co. .................. Elkins West Charleston Feed Co. Charleston 
AN ACT concerning Commercial Fertilizers, and repealing chapter seventy-two of the Acts of the Legislature of West Virginia, of eighteen hundred and ninety-one.

\section{(Passed February 2, 1901.)}

Be it enacted by the Legislature of West Virginia:

I. Every person who shall sell, offer or expose for sale, or have in his possession with intent to sell in this State, any commercial fertilizer or manure, shall affix conspicuously to every package thereof a plainly printed statement, clearly and truly certifying the number of net pounds of fertilizer in a package, the name, brand or trade-mark under which the fertilizer is sold, the name and address of the manufacturer, the place of manufacture, and stating the percentage of nitrogen or its equivalent in ammonia; of potash, soluble in distilled water; and of phosphoric acid in available form, soluble in distilled water, reverted, as well as the total phosphoric acid, and the materials from which said constituents are derived. In the case of those fertilizers which consist of other cheaper materials, said labels shall give correct general statement of the composition and ingredients of the elements relied upon, contained in the fertilizer which it accompanies.

2. Before any commercial fertilizer is sold, offered or exposed for sale in this State, the manufacturer, importer or party who causes it to be sold, exposed or offered for sale, shall file with the Director of the West Virginia Agricultural Experiment Station, under oath, the statement required to be affixed under section one of this act.

Provided, That when the manufacturer of any brand of fertilizer or manure shall file said sworn statement, no agent or dealer shall be required to file such statement. The making of an affidavit required by this chapter falsely shall be perjury.

3. The manufacturer, importer, agent or seller of any brand of commercial fertilizer or material used for manurial pur- 
poses, shall pay for each brand at the time he files a sworn statement required by section two of this act a registration fee of one dollar per annum.

4. The registration fee required to be paid by section three of this act, shall be paid to the treasurer of the West Virginia University for the use of the Agricultural Experiment Station, and the party making such payment shall take from the said treasurer triplicate receipts therefor, one of which he shall retain and the other shall be deposited, one with the Director of the Agricultural Experiment Station, and the other with the Secretary of the Board of Regents, and by them filed and preserved in their respective offices.

5. Immediately after the filing of the receipts aforesaid, with the Director of the Agricultural Experiment Station, said Director shall issue a certificate to the party making such payment, stating the amount of fees paid, and the name, brand or trade-mark under which the fertilizer is sold, the name and address of the manufacturer or importer, the place of manufacture, and that the applicant for said certificate is authorized to sell said fertilizer within the State of West Virginia for the period of one year from the first day of January to the thirty-first day of December, inclusive. Said certificates may be issued at any time for and during the current year, and may be issued during the month of December for the year commencing on the first day of January thereafter.

6. It shall be the duty of the Director of the West Virginia Agricultural Experiment Station to print or cause to be printed a label or tag setting forth the sworn statement required in section two of this act. The Director shall furnish such labels or tags in quantities in one hundred or multiples thereof, to any person complying with the requirements of this act, and desiring to sell, offer or expose for sale any commercial fertilizer in this State, and shall receive pay therefor at the rate of forty cents for such number of labels or tags as may be required to be affixed to one ton of fertilizer, and shall, without delay, pay the same to the treasurer of the West Virginia University, for use of the Agricultural Experiment Station, and take duplicate receipts therefor, 
one of which he shall retain, and the other he shall deliver to the Secretary of the Board of Regents, who shall file and preserve the same in his office.

7. The Board of Regents of the West Virginia University shall expend the money received under the provision of this act in meeting the legitimate expenses of the Station in making analysis of fertilizer, in experimental tests of same, and in such other experimental work and purchases as shall inure to the benefit of the farmers of this State, and shall include in their annual report a statement of the receipts and disbursements thereof.

8. It shall be the duty of the Director of the said Experiment Station, in person or by deputy, to take samples for analysis from any lot or packages of any fertilizer or manure which may be in the possession of any dealer in the State, and he is hereby authorized to prescribe and enforce such rules and regulations as he may deem necessary to carry fully into effect the true intent and meaning of this act; and any agriculturist, purchaser of any fertilizer or manure in this State may take a sample of the same under the rules and regulations of the Director of the said Experiment Station and forward the same to him for analysis, and if the Director has reason to believe that the manufacturer of, or dealer in, such fertilizer or manure has made any false or fraudulent representations in regard to said fertilizer or manure, he shall cause the said sample to be analyzed free of charge, and certify the same to the person forwarding the sample. And it shall be the duty of every agent and dealer in fertilizers or manures, within the meaning of this act, in this State, when ordering consignments thereof, to promptly notify the Director of the said Experiment Station of the amount crdered, and the place and approximate time of delivery of said fertilizer and manures.

9. Said Director shall also publish by bulletin, the brand, name and location of the manufacturer and chemical analysis of every fertilizer or manure analyzed or caused to be analyzed by him. Said last publication to be made, if practicable, before the time at which said fertilizer is to be applied to the soil.

Io. Any manufacturer or vendor of any fertilizer or manure who shall sell or offer or expose for sale any fertilizer 
or manure without having previously complied with the provisions of this act as herein before set forth, shall be deemed guilty of a misdemeanor, and upon conviction thereof shall be fined not less than fifty nor more than five hundred dollars.

II. Any company, firm or corporation who shall wilfully remove from or deface or change any label, or tag, or brand affixed to any package of fertilizer under the provisions of this act before such fertilizer has been used for manurial purposes, or who shall sell such fertilizer without such label or tag being affixed thereto at the time of the sale, shall be deemed guilty of a misdemeanor, and upon conviction thereof, shall be fined not less than ten nor more than fifty dollars for each offense.

12. Any company, firm of person who shall remove or cause to be removed from any package of commercial fertilizer or mannure any statement, label or tag affixed thereto under the provisions of this act, and affix or cause the same to be affixed to any other package of commercial fertilizer or manure, shall be deemed guilty of a misdemeanor, and upon conviction thereof shall be fined not less than ten nor more than fifty dollars for each offense.

13. Any company, firm or person violating any of the provisions of this act, or who fails to comply with any of the requirements of this act, shall be deemed guilty of a misdemeanor, and upon conviction thereof, shall, when no other penalty is prescribed, be fined not less than ten nor more than one hundred dollars for each offense. But this act shall not be construed to apply to any one who manufactures fertilizers for his own use and not for sale.

I4. The Director of said Experiment Station shall report to the prosecuting attorney of the county where the offense was committed all violations of the provisions of this act, and failure to comply therewith, and a copy of any label, statement or tag required to be filed with said Director or prepared by him, and any analysis made or caused to be made by him, when duly certified by said Director, shall be received in evidence in any prosecution or suit for any violation of the provisions of this act. 
I, Harry Shaw, Clerk of the House of Delegates of West Virginia, hereby certify that the paper hereto annexed is a true copy of an Act of the Legislature of West Virginia passed on the second day of February, I9or, and approved by the Governor on the ninth day of February, I90I ; and that the same takes effect ninety days from the date of its passage.

Given under my hand this March 20th, I90I.

HARRY SHAW, Clerk of the House of Delegates. 
THE FOLLOWING IS A LIST OF THE MANUFACTURERS, AND THE NAMES OF THE BRANDS OF FERTILIZERS REGISTERED FOR SALE IN WEST VIRGINIA FOR THE YEAR 1906.

\section{AMERICAN AGRICULTURAL CHEMICAL CO.,}

No. 26 Broadway, New York, and No. 208 North Calvert STREET, BALTIMORE. Bone.

A. A. C. Co.'s Pure Ground A. A. C. Co.'s Fine Ground Bone.

A. A. C. Co.'s Bone Meal.

A. A. C. Co.'s Genuine German Kainit.

A. A. C. Co.'s Gem Alkaline Prosphate.

A. A. C. Co.'s Big Crop Phos-' phate.

A. A. C. Co.'s Regular Corn Fertilizer.

A. A. C. Co.'s Superphosphate.
A. A. C. Co.'s Kainit.

Bradley's Bean and Potato.

Bradley's Niagara Phosphate.

Bradley's Alkaline Bone.

Bradley's Soluble Dissolved Bone.

Bradley's Dissolved Bone With Potash.

Canton Chemical Game
Guano. nure.

Canton Chemical Potato Ma-

Canton Chemical Bakers' Special Wheat, Corn and Grass Manure.

Canton Chemical Harrow Brand Crop Grower.

Canton Chemical Eagle Phosphate.

Canton Chemical Soluble Alkaline Bone.

Canton Chemical Soluble Bone and Potash.
Canton Chemical Baker's Dissolved S. C. Bone.

Canton Chemical Resurgam Guano.

Canton Chemical Canton Patron's Bone Mixture.

Cleveland Dryer Forest City Buckeye.

Cleveland Dryer Horsehead Phosphate and Potash.

Cleveland Dryer XXX Phosphate.

Cleveland Dryer Horsehead Phosphate.

Bone.

Detrick's Dissolved S. C.

Detrick's Quickstep Bone Phosphate.

Detrick's Kangaroo Komplete Kompound.

Detrick's. Standard Potash Fertilizer.

Detrick's Corn and Oats Fertilizer.

Detrick's Imperial Compound.

Detrick's Paragon Ammoni.* ated Bone, Phospnate and Potash.

Detrick's Soluble Bone, Phosphate and Potash.

Great Eastern Unammoniated Wheat Special.

Great Eastern Dissolved Bone.

Great Eastern Japanese Wheat Grower. 
Great Eastern Soluble Bone and Potash.

Great Eastern High Grade Bone and Potash.

Great Eastern English Wheat Grower.

Great Eastern Wheat Special.

Great Eastern Vegetable, Vine and Tobacco.

Great Eastern Corn Fertilizer.

Lazaretto Dissolved Bone and Potash.

Lazaretto Special Potato and Tobacco Fertilizer.

Lazaretto Bone Compound.

Lazaretto Dissolved Bone Phosphate.

Lazaretto High Grade Dissolved Bone and Potash

Lazaretto Ammoniated Bone Phosphate.

Maryland O. K. Ammoniated.

Maryland Ammoniated Bone.

Maryland Tornado Fertilizer.

Maryland Bono-Superphosphate.

Maryland Dissolved S. C. Bone.

Pacific Dissolved Bone Phosphate.

Pacific Dissolved Bone and Potash.

Pacific Potato Phosphate.

Pacific Nobsque Guano.

Pacific A No. 1 Phosphate.

Reese's Half and Half.

Reese's High Grade Potash

Mixture.

Reese's Elm Phosphate.

Susquehanna Superior Rock Phosphate

Susquehanna Alkaline Bone.

Susquehanna High Grade Bone and Potash.

Susquehanna Ammoniated Bone Phosphate.
Susquehanna XXV Phosphate.

Susquehanna Crop Grower.

Southern Fertilizer Co.'s Ammoniated Dissolved Bone.

Southern Fertilizer Co.'s

Farmers' Choice.

Southern Fertilizer Co.'s General Crop Grower.

Southern Fertilizer Co.'s Farmers' Mixture.

Southern Fertilizer Co.'s

Dissolved Bone Phosphate.

Southern Fertilizer Co.'s Pure Ground Bone.

Washington \& Alexander's Regular Corn Mixture.

Wheeler's Corn Fertilizer.

Wheeler's Potato Manure.

Wheeler's Royal Wheat

Grower.

Wheeler's Wheat and Clover Fertilizer.

Wheeler's Electrical Dissolved Bone.

Wheeler's Unammoniated Wheat Grower.

Williams \& Clark's Good Grower Potato Phosphate.

Williams \& Clark's Royal Bone Phosphats.

Williams \& Clark's Prolific Crop Producer.

Williams \& Clark's Dissolved Bone and Potash.

Williams \& Clark's Acorn Acid Phosphate.

Zell's Ammoniated Bone Super Phosphate.

Zell's Economizer Phosphate.

Zell's Little Giant.

Zell's Electric Phosphate.

Zell's Dissolved Bone Phosphate.

Zell's Dissolved S. C. Phosphate.

Zell's Special Compound for Potatoes and Vegetables. 


\section{ARMOUR FERTILIZER WORKS,}

Baltimore, MD.

Genuine German Kainit.

Fruit and Root Crop Special.

Phosphate and Potash No. 1.

Cereal Phosphate.

Grain Grower.

Bone Meal.

Wheat Special. ial.

Wheat Corn and Oats Spec-
Star Phosphate.

Phosphate and Potash No. 2.

Ammoniated Bone With Potash.

Raw Bone Meal.

High Grade Potato.

Royal Ammoniated Bone.

Bone Blood Potash.

\section{BALTIMORE PULVERIZING CO.,}

BALTIMORE, MD.

Farmers' Favorite Fertilizer. Special Fall Mixture.

BAUGH \& SONS CO.,

BALTIMORE, MD. er.

Baugh's General Crop Grow- | Baugh's Pure Dissolved Ani-

Baugh's Ammoniated Soluble Alkaline.

Baugh's Soluble Alkaline.

Lobos Peruvian Guano.

Baugh's Special Potato Manure ( $10 \%$ Potash).

Baugh's Potato and Truck Special.

Baugh's High Grade Acid Phosphate Rock.

Baugh's High Grade Potash Mixture.

B'augh's Pure Bone Meal. mal Bone.

Baugh's Export Bone With Potash.

Baugh's Animal Bone and Potash Compound.

Baugh's Complete Animal Bone Fertilizer.

Baugh's Excelsior Corn and Peanut Guano.

Baugh's Double Eagle \$25. Phosphate and a Raw Bone Super Phos.

Baugh's Peninsula Grain Producer.

\section{BOWKER FERTILIZER CO., Cincinnati, Ohio.}

Bowker's Hill and Drill Phos. Bowker's With Potash.

Bowker's Sure Crop.

Bowker's Dissolved Bone With Potash.

Bowker's Potash Fertilizer.

Bowker's Harvest Bone.
Bowker's 10\% Manure.

Bowker's Bone Meal.

Bowker's Dissolved Bone Phosphate.

Bowker's Super Phosphate With Potash for Grain and Grass.

Bowker's Soluble Bone. 


\section{THE BRYANT FERTILIZER CO.,}

ALEXANDER, VA. Bone.

Bryant's S. C. Dissolved

\section{CHICAGO FERTILIZER CO.,} Chicago, ILI.
No. 1 Acid Phosphate. Diamond Phosphate. Calumet Phosphate. Wheat and Corn Special.
Bryant's Queen Ammd. Dissolved Bone.

Bryant's Formula “E."

\section{W. S. FARMER \& CO., \\ BALTIMORE, MD.}

Standard Fertilizer.

B. \& P. Mixture.

Harvest Queen.
Western Bone Black and Potash.

Bone, Blood and Potash.

Potato Special.

Chicago Bone Meal.

\section{GRIFFITH \& BOYD.}

BALTIMORE, MD.

Harvest Queen Phosphate.

Valley Fertilizer.

Special Grain Grower.

High Grade Acid Phosphate.
Dissolved Raw Bone.

Dissolved S. C. Bone.

\section{S. M. HESS \& BRO.,}

\section{Philadel,PHIA, PA.}

Keystone Bone Phosphate. Soluble Bone and Potash. Ëmperor Phosphate.
Soluble Bone. Wheat and Grass Manure. 


\section{P. HUBBARD \& CO., \\ BALTIMORE, MD.}

Hubbard's Old Economy.

\section{THE JARECKI CHEMICAL CO.,}

Sandusky and Cincinnati, OHio.

No. 1 Fish Guano.

Ground Bone.

Square Brand Phosphate and Potash.
Fish and Potash, Tobacco and Potato Food.

Dissolved Bone With Potash.

THE MARIETTA BONE AND PHOSPHATE CO., MARIETTA, OHIO.

Pure Raw Bone.

Potato and Truck Special. Buckeye.
Corn, Oats and Wheat Grower.

Acld Phosphate.

\section{MUSKINGUM VALIEY FERTILIZER CO., MARIETTA, OHIO.}

Peerless Brand Special Vegetable Grower.

Peerless Brand Acidulated Bone Phosphate.
Peerless Brand Acld Phoo phate. Peerless Brand Bone Meal.

\section{B. MARTIN CO.,}

PHILADELLPHIA, BALTIMORE, NORFOLK.

Martin's Sure Ground. Martin's Dissolved Organic Compound.
Martin's Animal 6\% Tankage. 


\section{G. OBER \& SONS CO.,}

BALTIMORE, MD.

Ober's Dissolved Animal
Bone.
Ober's Farmers' Standard
Ammoniated Phosphate.
Ober's Farmers' Mixture.
Ober's Independent Ammo.
Super Phosphate.
Ober's Dissolved Bone, Phos-
phate and Potash.
Ober's Dissolved Bone Phos-
phate.

Ober's High Grade Acid Phosphate.

Ober's Standard Potash Compound.

Special Mixture for Wheat.

S. \& S. Special Wheat Compound.

Langdun Mixture.

Pure Raw Bone Meal.

Pure Bone Meal.

\section{OHIO FARMERS FERTILIZER CO., Columbus, Оhiо.}

Superior Phosphate.

Alkaline Bone.

Soluble Bone and Potash.

General Crop Fish Guano. Down.

Wheat Malrer and Seeding

Corn, Oats and Wheat Fish Guano.

Potato and Tobacco Special.

Fine Ground Bone Meal.

\section{PIEDMONT MT. AIRY GUANO CO.,}

\section{BALTIMORE, MD.}

Piedmont Royal Ammoniated Bone and Potash.

Piedmont Dissolved Phosphate Potash Goods.

Piedmont Potato Producer.

Piedmont Farmers' High Grade Bone and Potash.

Pure Raw Bone Meal.

Piedmont Special Wheat and Grass Compound.

Piedmont High Grade Ammoniated Bone and Potash.

Piedmont P. G. Guano.

Piedmont Plow Brand.

Piedmont Special Potash Mixture.
Piedmont Soluble Bone and Potash.

Levering's Excelsior.

Levering's Harvest Queen.

Piedmont Farmers' Favorite. Piedmont Harvest King.

General Crop Grower.

Piedmont Dissolved Animal Bone.

Levering's IXL Crop Grower. Piedmont $16 \%$ Acid Phosphate.

Piedmont Pure Raw Bone Mixture.

Piedmont High Grade S. C. B'one. 


\section{PITTSBURG PROVISION AND PACKING CO.,}

Pittssburg, Pa.

Pure Raw Bone Meal.

Pure Bone With Potash.

Corn and Potato Fertilizer. '

Keystone Fertilizer.

Crescent Butchers' Ground
Bone.

Guano Fertilizer.

Phosphate and Potash.

Acid Phosphate.

\section{RASIN-MONUMENTAL, CO.,}

BALTIMORE, MD.

Rasin's $16 \%$ Acid Phosphate. Rasin's Special Bone and Potash.

Langdun's Mixture.

Rasin's XXX Fertilizer.

Rasin's Empire Guano.

Rasin's Dissolved Bone.

Rasin's IXL Fertilizer.

William Penn Crop Grower.

Rasin's Bone and Potash Fertilizer.

Seawall Special.

Rasin's Acid Phosphate.

Rasin's Dissolved S. C. Phosphate.
Rasin's National Crop Compound.

Special Formula for Corn and Buclewheat.

Rasin's Special Fish and Potash Mixture.

Arundel Complete.

Victoria Bone.

Washington \& Alexander's Special Mixture for Wheat.

Rasin's Special Bone and Potash.

Wheat, Corn and Oats Mixture.

SWIFT \& CO.,

Chicago, Ili.

Swift's Superphosphate.

Complete Fertilizer.

Pure Raw Bone Meal and Potash.

Pure Raw Bone Meal.

Poor Bone Meal.
Potato and Tobacco Grower. Champion Wheat and Corn. Grower.

Pure Special Bone Meal.

Swift's Pure Bone Meal and Blood.

\section{P. THOMAS \& SON CO.,}

Philadeliphia, Pa.

S. C. Phosphate.

Alkaline Bone.

Special Alkaline Bone.

Special Corn Fertilizer.
Superior Super Phosphate.

Pure Ground Bone.

Thomas Wheat and Corn Fertilizer. 


\section{TUSCARORA FERTILIZER CO.,}

BAL,TIMORE, MD.

Cereal Phosphate. Acid Phosphate. Fruit and Potato. Tuscarora Alkaline. Tuscarora Big Four.
Special Crop Grower. Bone and Potash. Ammoniated Phosphate. Tuscarora Standard. Bone and Potash No. 2.

\section{THE WOOLDRIDGE ORCHILLA CO.,}

\section{BALTIMORE, MD.}

Orchilla Guano.

German Alkaline Mirture. Virginia Bell Potash Mixture.

Bizzy Bee.

Old Hickory.

\section{Fauitless.}

Tiger Bone Stock Phosphate. High Grade Potato and Tomato Grower.

\section{VIRGINIA-CAROLINA CHEMICAL CO.,}

\section{BALTIMORE, MD.}

V. C. C. Co. Standard Crop Grower.

V. C. C. Co. Special Compound for Wheat.

V. C. C. Co. Corn and Buckwheat Special.

V. C. C. Co. Special Bone and Potash.

V. C. C. Co. Standard Bone and Potash.

V. C. C. Co. $14 \%$ Acid Phosphate.

V. C. C. Co. Royal Acld Phosphate.

V. C. C. Co. Fish, Bone and Potash.

V. C. C. Co. Special Potash Mixture.

Casler's Wheat and Grass Compound.

Cooper's Special Wheat
Allison \& Addison's Star Brand Guano.

Allison \& Addison's Little Giant Grain and Grass Grower. Allison \& Addison's B. P. Potash Mixture.

Allison \& Addison's Standard Acid Phosphate.

S. W. Travers \& Co.'s Beef, Blood and Bone Fertilizer.

S. W. Travers \& Co.'s Champion Corn, Wheat and Grass Grower.

S. W. Travers \& Co.'s Capital Bone and Potash Compound.

S. W. Travers \& Co.'s Dissolved Bone Phosphate.

S. W. Travers \& Co.'s Capltal Dissolved S. C. Bone.

Southern Chem. Co.'s Sun Brand Guano. 


\section{ROBER'T A. WOOLDRIDGE CO.,}

\section{BALTIMORE, MD.}

Florida Acid Phosphate. German Potash Mixture. Liberty Bell Potash Mixture. Old Sledge Potash Mixture.
Champion Giant Phosphate. Chieftain Phosphate.

Triumph Phosphate. Special Potato Fertilizer.

T. W. WOOD \& SONS,

RICHMOND, VA.

Standard Potato.

Standard Vegetable. Standard Corn.
Standard Grain and Grass. Acld Phosphate. Wood's Pure Animal Bone.

Application for bulletins of this Station should be addressed to the Director of the West Virginia Agricultural Experiment Station, Morgantown, W. Va.

(The bulletins named below are available for distribution.)

Bulletin No. 12.

The Canada Thistle, by C. F. Millspaugh, M. D., December 1890.

Bulletin No. 19.

Your Weeds and Your Neighbors, Part I. Weeds as Fertil-

Bulletin No. $\mathbf{3 3}$. izers, by C. F. Millspaugh November 1891.

Sub-Irrigation in the Greenhouse, by Wm. F. Rane, September 1893.

Bulletin No. 34.

Fertilizer Analysis for the Year Ending December 31, 1893, by J. J. DeRoode, December 1893 .

Bulletin No. 37.

Electro Horticulture.

Incandescent Lamps.

Bulletin No, 38.

By F. Wm. Rane, July 1894.

Potato Blight. Potato Scab, by F. Wm. Rane, November 1894.

Bulletin No. 39.

Vegetables, by F. Wm. Rane, January 1895.

Bulletin No. 40.

Commercial Fertilizers, by B. H. Hite, December 1895. 
No. 2, Special Bulletin.

Proceedings Connected with the Celebration upon the Completion of the Station Building and the Organization of the Sheep Breeders' \& Woolgrowers' Association, April 5, 1894.

Bulletin No. 54.

Nursery Hints, by L. C. Corbett.

\section{Bulletin No. 55.}

Sugar Beet Investigation in 1898 , by J. H. Stewart and B. H. Hite, 1899.

\section{Bulletin No. 56 .}

Report on Investigation to Determine the Cause of Unhealthy Condition of Spruce and Pine from 1880 to 1893 , by A. D. Hopkins.

Bulletin No. $\mathbf{5 7}$.

Commercial Fertilizers, by B. H. Hite and T. F. Watson, 1899.

Bulletin No. 58.

The Effect of Pressure in the Preservation of Milk, by B. H. Hite, 1899.

Bulletin No. 59.

Whole Corn Compared with Corn Meal for Fattening Hogs, by J. H. Stewart and Horace Atwood, 1899.

Bulletin No. 60 .

Poultry Experiments, by J. H. Stewart and Horace Atwood, 1899.

Bulletin No. 61.

Sheep Feeding Experiments, by J. H. Stewart and Horace Atwood, 1899.

Bulletin No. 62.

A Study of the Effect of Incandescent Gas-Light on Plant Growth, by L. C. Corbett, 1899.

Bulletin No. 64.

Sugar Beet Investigation in 1899 , by J. H. Stewart and B. H. Hite, 1900 .

Bulletin No. 66.

Fruit Diseases and How to Treat Them, by L. C. Corbett, 1900 .

Bulletin No. 67.

The Hessian Fly in West Virginia and How to Prevent Losses From Its Ravages, by A. D. Hopkins, 1900 .

Bulletin No. 68 .

The Periodical Cicada or Seventeen-Year Locust in W. Va., by A. D. Hopkins, 1900.

Bulletin No. 69.

Report on Examination of Wheat Stubble from Different Sections of the State, by A. D. Hopkins, 1900.

Bulletin No. 70.

Spraying, by L. C. Corbett, 1900.

Bulletin No. 71 .

Poultry Experiments, by J. H. Stewart and Horace Atwood, 1900.

Bulletin No. 73.

Poultry Experiments, Loss of Weight in Eggs During Incubation, by J. H. Stewart and H. Atwood, 1901.

Bulletin No. 74.

Cold Storage, L. C. Corbett, 1901. 
Bulletin No. 75.

Apple Districts of W. Va., L. C. Corbett, 1901.

Bulletin No. 76 .

Commercial Fertilizers, B. H. Hite and Horace Atwood, 1901.

Bulletin No. 77.

The New Fertilizer Law, J. H. Stewart, 1901.

Bulletin No. 78 .

Treatment of San Jose Scale, by J. H. Stewart, 1902.

Bulletin No. 84.

Experiments with Buckwheat and Oats, J. H. Stewart and Horace Atwood, 1903.

Bulletin No. 86.

Cranberries in West Virginia, L. C. Corbett, 1903.

Bulletin No. 87 .

Greenhouses, L. C. Corbett, 1903 .

Bulletin No. 88.

Poultry Experiments, J. H. Stewart and Horace Atwood, 1903.

Bulletin No. 89.

Rural Water Supply, C. D. Howard, 1903.

Bulletin No. 90.

Sheep Feeding Experiments, by J. H. Stewart and Horace Atwood, October 1903.

Bulletin No. 92.

Commercial Fertilizers, by J. H. Stewart and B. H. Hite, December 1903.

Bulletin No. 93.

Mixtures and Appliances for Spraying, by T. C. Johnson, November 1903.

Bulletin No. 94.

Diseases of Melons and Cucumbers, by John L. Sheldon, December 1904 .

Bulletin No. 95.

Commercial Fertilizers, by J. H. Stewart and B. H. Hite,

Bulletin No. 97. December 1904.

Commercial Fertilizers, by J. H. Stewart and B. H. Hite,

Bulletin No. 98. December 31, 1905.

Bulletin No. 99.

Raising Chicks Artificially, by J. H. Stewart and Horace Atwood, January 30,1906 .

Experiments with Fertilizers, by J. H. Stewart and Horace Atwood, February 1, 1906.

Bulletin No. 100.

The Grape Curculio, by Fred E. Brooks, March 1906.

Bulletin No. 101.

Experiments in the Manuring of a Meadow, by J. H. Stewart

Bulletin No. 102. and Horace Atwood, March 1906.

Poultry Experiments, by J. H. Stewart and Horace Atwood, May 1906.

Bulletin No. 103.

Occurrence of Barium in the Ohio Valley Brines and its Relation to Stock-Poisoning, by C. D. Howard, June 1906. 
Bulletin No. 105.

Tubercles on Legumes With and Without Cultures, by John L. Sheldon, June 1906.

Bulletin No. 106.

Feeding Experiments with Milch Cows, by J. H. Stewart and Horace Atwood, June 1906.

Bulletin No. 107.

A Test of Different Sprays for the San Jose Scale, Entomological Department, June 1906.

Special Bulletin No. 3.

San Jose Scale in West Virginia, Entomological Department, March 1905. 

